\title{
SKYSCRAPER POLYTOPES AND REALIZATIONS OF PLANE TRIANGULATIONS
}

\author{
IGOR PAK* AND STEDMAN WILSON ${ }^{\dagger}$
}

\begin{abstract}
We give a new proof of Steinitz's classical theorem in the case of plane triangulations, which allows us to obtain a new general bound on the grid size of the simplicial polytope realizing a given triangulation, subexponential in a number of special cases.

Formally, we prove that every plane triangulation $G$ with $n$ vertices can be embedded in $\mathbb{R}^{2}$ in such a way that it is the vertical projection of a convex polyhedral surface. We show that the vertices of this surface may be placed in a $4 n^{3} \times 8 n^{5} \times \zeta(n)$ integer grid, where $\zeta(n) \leq\left(500 n^{8}\right)^{\tau(G)}$ and $\tau(G)$ denotes the shedding diameter of $G$, a quantity defined in the paper.
\end{abstract}

\section{INTRODUCTION}

Steinitz's theorem states every 3-connected plane graph $G$ is the graph of a 3-dimensional convex polytope. An important corollary of the original proof is that the vertices of the polytope can be made integers. The quantitative Steinitz problem $[\mathrm{R}]$ asks for the smallest size of such integers as they depend on a graph. The best current bounds are exponential in the number of vertices in all three dimensions, even when restricted to triangulations, see [RRS]. A variant of these bounds, in terms of bit complexity, appears in [DG], in which the authors demonstrate that 3-connected planar triangulations can be realized as convex 3 -polyhedra whose vertices may be represented using a polynomial number of bits (see [DG], Theorem 2.1).

In this paper we improve these bounds in two directions. While the main result of this paper is rather technical (Theorem 4.2), the following corollary requires no background.

Corollary 1.1 Let $G$ be a plane triangulation with $n$ vertices. Then $G$ is a graph of a convex polyhedron with vertices lying in a $4 n^{3} \times 8 n^{5} \times\left(500 n^{8}\right)^{n}$ integer grid.

This result improves known bounds in two directions at the expense of a somewhat weaker bound in the third direction. We mention that an improvement in one direction, at the expense of the other two, is already given by Schultz, who presents an embedding of general 3-polytopes in an integer grid that is polynomial (in fact linear) in one dimension, but superexponential in the other two (see [S], Theorem 3). We call simplicial polytopes obtained from Corollary 1.1 "skyscraper polytopes", as they are small (polynomial in size) in two directions but generally have superexponential size in the third. However, for large families of graphs we make sharp improvements in the third direction as well. Below we give our our main application.

Date: August 15, 2015.

*Department of Mathematics, UCLA, Los Angeles, CA 90095, USA; \{pak\}@math.ucla.edu.

${ }^{\dagger}$ Department of Mathematics, Ben Gurion University, Be’er Sheva, Israel; \{stedmanw\}@gmail.com. 
A grid triangulation of $[a \times b]=\{1, \ldots, a\} \times\{1, \ldots, b\}$ is a triangulation with all grid points as the set of vertices. These triangulations have a curious structure, and have been studied and enumerated in a number of papers (see [A, KZ, We] and references therein).

Corollary 1.2 Let $G$ be a grid triangulation of $[k \times k]$, such that every edge sits in an $\ell \times \ell$ subgrid. Then $G$ is a graph of a convex polyhedron with vertices lying in a $O\left(k^{6}\right) \times O\left(k^{10}\right) \times$ $k^{O(\ell k)}$ integer grid.

Setting $\ell=O(1)$ as $k \rightarrow \infty$, for the grid triangulations as in the corollary, we have a subexponential grid size in the number $n=k^{2}$ of vertices: $O\left(n^{3}\right) \times O\left(n^{5}\right) \times \exp O(\sqrt{n} \log n)$.

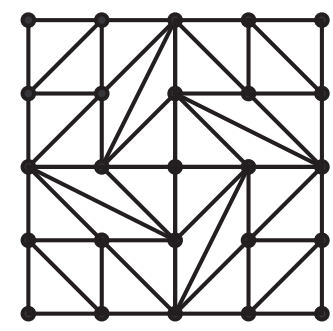

FiguRE 1. An example of a grid triangulation of $[5 \times 5]$, with $\ell=3$.

The basic idea behind the best known bounds in the quantitative Steinitz problem is as follows (see [R, RRS, Ro]). Start with a Tutte spring embedding of $G$ with unit weights [T], and lift it up to a convex surface according to the Maxwell-Cremona theorem (see $[\mathrm{L}, \mathrm{R}]$ ). Since Tutte's embedding and the lifting are given by rational equations, this embedding can be scaled to an integer embedding. However, there is only so much room for this method to work, and since the determinants are given by the number of spanning trees in $G$, the bounds cannot be made subexponential in the case of triangulations.

There are several interesting proofs of the Steinitz theorem [Z1, Z2] and some simplified versions of the proof for the special case of triangulations $[D G, G]$. However, none seem to suggest a way to substantially decrease the size of the integer grid in the case of triangulations. The proof we present follows a similar idea, based on lifting a plane graph, but in place of the Tutte spring embedding we present an inductive construction. In essence, we construct a strongly convex embedding of plane triangulations, based on a standard inductive proof of Fáry's theorem [F]. We make our construction quantitative, by doing this on a $O\left(n^{3}\right) \times O\left(n^{5}\right)$ grid, thus proving a result reminiscent of the main result in [BR]. The key difference is that, while our construction uses a larger grid size than that in $[\mathrm{BR}]$, it produces a drawing with a convex boundary at each step of the construction.

This step by step convexity allows us to lift the resulting triangulation directly to a convex surface. The inductive argument allows us to obtain a new type of quantitative bound $\zeta(n)=n^{O(\tau(G))}$ on the height of the lifting. The parameter $\tau(G)$ here may be linear in $n$ in the worst case. However, this parameter $\tau(G)$ is sublinear in a number of special cases, such as the grid triangulations mentioned above, where $\tau(G)=\Theta(\sqrt{n})$ (see §6.5). In fact, this is this lower bound. Indeed, it is easy to show that

$$
\tau(G) \geq \operatorname{diam}(G), \operatorname{diam}\left(G^{\prime}\right),
$$

which implies that $\tau(G)=\Omega(\sqrt{n})$ for 3-connected plane triangulations $G$. 
The rest of this paper is structured as follows. In the next section we recall some definitions and basic results on graph drawing. In Section 3.3 we prove Theorem 3.3, the crucial technical result on graph embedding. Then, in Section 4, we define the shedding diameter and prove Theorem 4.2, the main result of this paper. We discuss grid triangulations in Section 5, and conclude with final remarks in Section 6.

\section{Definitions AND BASIC RESUlts}

Let $G=(V, E)$ denote a plane graph. By abuse of notation we will identify $G$ with the subset of $\mathbb{R}^{2}$ consisting of its vertices and edges. We write $V(G)$ for the vertices of $G$ and $E(G)$ for the edges of $G$. When $G$ is 2-connected we let $\mathcal{F}(G)=\left\{F_{1}, \ldots, F_{m}\right\}$ denote the set of (closed) bounded faces of $G$. We define $\mathbf{F}(G)=\bigcup_{i} F_{i}$, the region of $\mathbb{R}^{2}$ determined by $G$. For a subgraph $H$ of $G$, we write $H \subseteq G$.

When $G$ is 2-connected, a vertex $v \in V$ is called a boundary vertex if $v$ is in the boundary of $\mathbf{F}(G)$, and an interior vertex otherwise. Similarly, an edge $e \in E$ is called a boundary edge if $e$ is completely contained in the boundary of $\mathbf{F}(G)$, and an interior edge otherwise. A diagonal of $G$ is an interior edge whose endpoints are boundary vertices of $G$. For a plane graph $G$ with vertex $v$, let $G-\{v\}$ denote the plane graph obtained by removing $v$ and all edges adjacent to $v$.

We say that two plane graphs $G, G^{\prime}$ are face isomorphic, written $G \sim G^{\prime}$, if there is a graph isomorphism $\psi: V(G) \rightarrow V\left(G^{\prime}\right)$ that also induces a bijection $\psi_{\mathcal{F}}: \mathcal{F}(G) \rightarrow \mathcal{F}\left(G^{\prime}\right)$ of the bounded faces of $G$ and $G^{\prime}$. This last property means that $v_{1}, \ldots, v_{k}$ are the vertices of a face $F \in \mathcal{F}(G)$ if and only if $\psi\left(v_{1}\right), \ldots, \psi\left(v_{k}\right)$ are the vertices of a face $F^{\prime} \in \mathcal{F}\left(G^{\prime}\right)$. By definition, $G \sim G^{\prime}$ implies that $G$ and $G^{\prime}$ are isomorphic as abstract graphs, but the converse in not always true. When $G \sim G^{\prime}$ and $v$ is a vertex of $G$, we will write $v^{\prime}$ for the corresponding vertex of $G^{\prime}$, indicating that a face isomorphism $\psi$ is defined by $v^{\prime}=\psi(v)$.

A geometric plane graph is a plane graph for which each edge is a straight line segment. A geometric embedding of a plane graph $G$ in the set $S \subseteq \mathbb{R}^{2}$ is a geometric plane graph $G^{\prime}$ such that $G \sim G^{\prime}$ and every vertex of $G^{\prime}$ is a point of $S$. For a point $u=(a, b) \in \mathbb{R}^{2}$, we will write $x(u)=a$ and $y(u)=b$ for the standard projections.

For a plane graph $G$ with $n$ vertices and an ordering of the vertices $\mathbf{a}=\left(a_{1}, \ldots, a_{n}\right)$, we define a sequence of plane graphs $G_{0}(\mathbf{a}), \ldots, G_{n}(\mathbf{a})$ recursively by $G_{n}(\mathbf{a})=G$ and $G_{i-1}(\mathbf{a})=$ $G_{i}(\mathbf{a})-\left\{a_{i}\right\}$. We will write $G_{i}$ for $G_{i}(\mathbf{a})$ when $\mathbf{a}$ is understood. If $v$ is a vertex of $G_{i}$ then we let $d_{i}(v)$ denote the degree of $v$ in the graph $G_{i}$.

A plane triangulation is a 2-connected plane graph $G$ such that each bounded face of $G$ has exactly 3 vertices. Note in particular that if $G$ is a plane triangulation then $\mathbf{F}(G)$ is homeomorphic to a 2-ball. A boundary vertex $v$ of a plane triangulation $G$ is a shedding vertex of $G$ if $G-\{v\}$ is a plane triangulation. Let $G$ be a plane triangulation with $n$ vertices. A vertex sequence $\mathbf{a}=\left(a_{1}, \ldots, a_{n}\right)$ is called a shedding sequence for $G$ if $a_{i}$ is a shedding vertex of $G_{i}(\mathbf{a})$ for all $i=4, \ldots, n$. We have the following technical lemma given in $[\mathrm{FPP}, \S 2]$, where it was used for an effective embedding of graphs.

Lemma 2.1 ([FPP]) Let $G$ be a plane triangulation. Then, for every boundary edge uv of $G$, there is a shedding sequence $\boldsymbol{a}=\left(a_{1}, \ldots, a_{n}\right)$ for $G$, such that $u=a_{1}$ and $v=a_{2}$.

In order to carry out the embeddings described below, we will need to strengthen the notion of a shedding sequence, so that each region $\mathbf{F}\left(G_{i}(\mathbf{a})\right)$ is convex, in a certain strong 
sense. We say that a strictly convex polygon $P \subset \mathbb{R}^{2}$ with designated edge $a_{1} a_{2}$ is projectively convex (with respect to $a_{1} a_{2}$ ) if $P$ is contained in the upper half-plane, $a_{1} a_{2}$ lies on the $x$-axis, and $a_{1}$ and $a_{2}$ are the unique leftmost and rightmost points of $P$, respectively. This last condition is the most notable, as the first two conditions may be obtained for any strictly convex polygon via an affine transformation (the last condition may then be obtained via a projective transformation, which motivated the terminology). A shedding sequence $\mathbf{a}=\left(a_{1}, \ldots, a_{n}\right)$ for a geometric plane triangulation $G$ is a convex shedding sequence if the region $\mathbf{F}\left(G_{i}(\mathbf{a})\right)$ is a projectively convex polygon with respect to the edge $a_{1} a_{2}$ for all $i=3, \ldots, n$. A geometric embedding $G^{\prime}$ of $G$ is sequentially convex if $G^{\prime}$ has a convex shedding sequence.

\section{Drawing the triangulation on a GRID}

3.1. A Rational Embedding. First we address a much easier question: How does one obtain a sequentially convex embedding of $G$ in $\mathbb{Q}^{2}$ (that is, with vertex coordinates ratio$n a l)$ ? We describe a simple construction that produces such an embedding. The method used to accomplish this easier task will provide part of the motivation and intuition behind the more involved method we will use to obtain a polynomially sized embedding in $\mathbb{Z}^{2}$.

Theorem 3.1 Let $G$ be a plane triangulation with $n$ vertices and boundary edge uv, and let $\boldsymbol{a}=\left(a_{1}, \ldots, a_{n}\right)$ be a shedding sequence for $G$ with $u=a_{1}, v=a_{2}$. Then $G$ has $a$ geometric embedding $G^{\prime}$ in $\mathbb{Q}^{2}$, such that the corresponding sequence $\boldsymbol{a}^{\prime}=\left(a_{1}^{\prime}, \ldots, a_{n}^{\prime}\right)$ is a convex shedding sequence for $G^{\prime}$.

Proof. We proceed by induction on $n$. If $n=3$ then we may take the triangle with coordinates $a_{1}^{\prime}=(0,0), a_{2}^{\prime}=(2,0), a_{3}^{\prime}=(1,1)$ as a sequentially convex embedding of $G$ in $\mathbb{Q}^{2}$.

If $n>3$, then by the inductive hypothesis there is an embedding $G_{n-1}^{\prime}$ of $G_{n-1}$ in $\mathbb{Q}^{2}$ such that $\left(a_{1}^{\prime}, \ldots, a_{n-1}^{\prime}\right)$ is a convex shedding sequence for $G_{n-1}^{\prime}$. Let $w_{1}, \ldots, w_{k}$ denote the neighbors of $a_{n}$ in $G$, and let $w_{1}^{\prime}, \ldots, w_{k}^{\prime}$ denote the corresponding vertices of $G_{n-1}^{\prime}$, ordered from left to right. If $w_{1}^{\prime} \neq a_{1}^{\prime}$, then let $z_{1}^{\prime}$ denote the left boundary neighbor of $w_{1}^{\prime}$. Similarly, if $w_{k}^{\prime} \neq a_{2}^{\prime}$, let $z_{2}^{\prime}$ denote the right boundary neighbor of $w_{k}^{\prime}$.

For adjacent vertices $u$ and $v$, we will denote the slope of the edge $u v$ by $s(u v)$. Similarly, we will denote the slope of a line $\ell$ by $s(\ell)$. Consider the lines $\ell_{1}, \ell_{2}, \ell_{3}, \ell_{4}$ spanned by the edges $z_{1}^{\prime} w_{1}^{\prime}, w_{1}^{\prime} w_{2}^{\prime}, w_{k-1}^{\prime} w_{k}^{\prime}$, and $w_{k}^{\prime} z_{2}^{\prime}$, respectively. If $w_{1}^{\prime}=a_{1}^{\prime}$, we may take $\ell_{1}$ to be any non-vertical line passing through $a_{1}^{\prime}$, with slope satisfying $s\left(\ell_{1}\right)>s\left(\ell_{2}\right)$. Similarly, if $w_{k}^{\prime}=a_{2}^{\prime}$, we may take $\ell_{4}$ to be any non-vertical line passing through $v^{\prime}$, with slope satisfying $s\left(\ell_{3}\right)>s\left(\ell_{4}\right)$.

Let $A_{1}, A_{4} \subset \mathbb{R}^{2}$ denote the open half-planes below the lines $\ell_{1}$ and $\ell_{4}$, respectively, and let $A_{2}$ and $A_{3}$ denote the open half-planes above the lines $\ell_{2}$ and $\ell_{3}$, respectively. Since $\mathbf{F}\left(G_{n-1}^{\prime}\right)$ is projectively convex with respect to $a_{1}^{\prime} a_{2}^{\prime}$, the slopes of the lines $\ell_{i}$ must satisfy $s\left(\ell_{1}\right)>s\left(\ell_{2}\right)>s\left(\ell_{3}\right)>s\left(\ell_{4}\right)$. Thus the region $S=A_{1} \cap A_{2} \cap A_{3} \cap A_{4}$ is non-empty (see Figure 2). Since each set $A_{i}$ is open, the set $S$ is open, so we may choose a rational point in $S$, call it $a_{n}^{\prime}$. For each $j=1, \ldots, k$ add a straight line segment $e_{j}$ between $a_{n}^{\prime}$ and the vertex $w_{j}^{\prime}$. Since $a_{n}^{\prime}$ lies in the region above the lines $\ell_{2}$ and $\ell_{3}$, each line segment $e_{j}$ will intersect $G_{n-1}^{\prime}$ only in the vertex $w_{j}^{\prime}$.

Let $G^{\prime}$ denote the plane graph obtained from $G_{n-1}^{\prime}$ by adding the vertex $a_{n}^{\prime}$ and the edges $e_{j}$. Then $G^{\prime}$ is clearly a geometric embedding of $G_{n}=G$, such that each vertex 

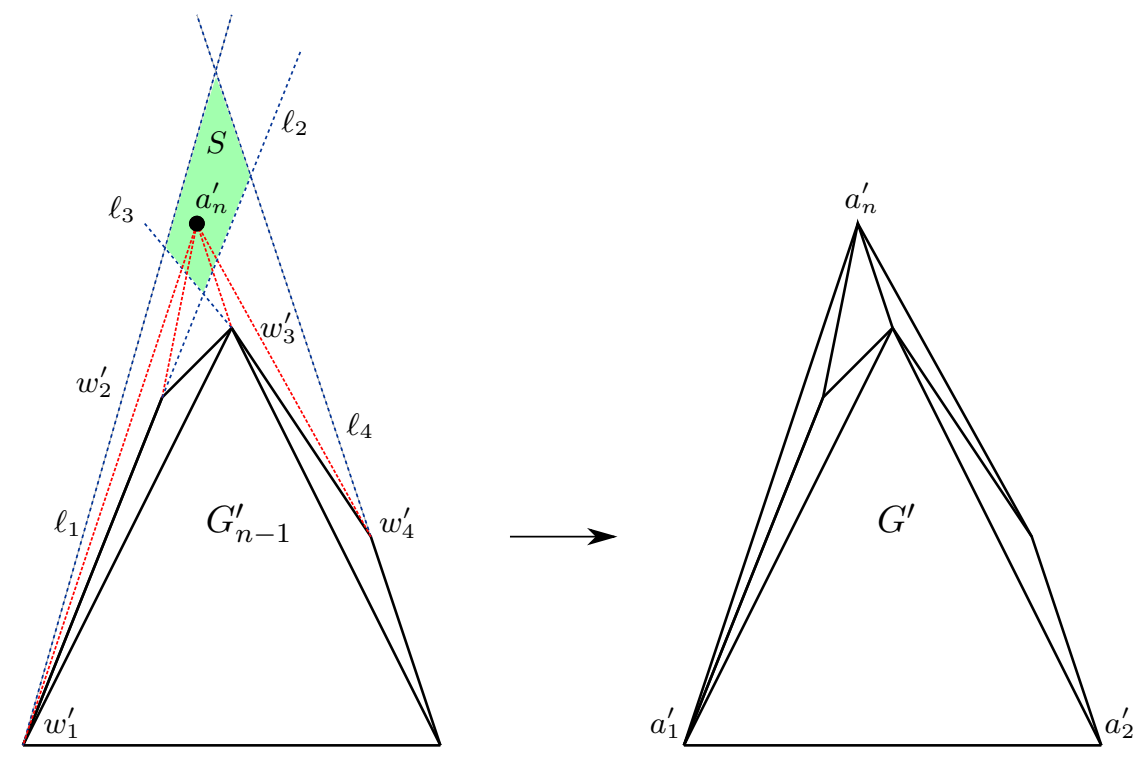

Figure 2. The new vertex $a_{n}^{\prime}$, chosen as a rational point of the set $S$.

$a_{i}$ corresponds to $a_{i}^{\prime}$, for $i=1, \ldots, n$. Furthermore, since $a_{n}^{\prime}$ lies in the region below the lines $\ell_{1}$ and $\ell_{4}$, the region $\mathbf{F}\left(G^{\prime}\right)$ is projectively convex with respect to $a_{1}^{\prime} a_{2}^{\prime}$. From this, together with the fact that $\left(a_{1}^{\prime}, \ldots, a_{n-1}^{\prime}\right)$ is a convex shedding sequence for $G_{n-1}^{\prime}$, we have that $\mathbf{a}^{\prime}=\left(a_{1}^{\prime}, \ldots, a_{n}^{\prime}\right)$ is a convex shedding sequence for $G^{\prime}$.

3.2. The Shedding Tree of a Plane Triangulation. Now we address the problem of embedding the triangulation $G$ on an integer grid. The idea behind our construction is roughly as follows. We start with a triangular base whose horizontal width is very large. We then show that, because this horizontal width is large enough, we may add each vertex in a manner similar to that used in the proof of Theorem 3.1, and we will always have enough room to find an acceptable integer coordinate. The crucial part of the construction is the careful method in which we add each new vertex. In particular, there are two distinct methods for adding the new vertex $a_{i}$, depending on whether $d_{i}\left(a_{i}\right)=2$ or $d_{i}\left(a_{i}\right)>2$. To facilitate the proper placement of the vertices $a_{i}$ with $d_{i}\left(a_{i}\right)=2$, we will appeal to a certain tree structure determined by the shedding sequence $\mathbf{a}$. We introduce the following definitions.

Let $G$ be a plane triangulation with shedding sequence a. We may assume that $G$ is embedded geometrically and is sequentially convex with respect to a (i.e. $G$ is embedded as in Theorem 3.1). Proceeding recursively, we will define a binary tree $T=T(G$, a), such that the nodes of $T$ are edges of $G$, and the edges of $T$ correspond to faces of $G$. We will consider all binary trees to be ordered. That is, we assume a fixed left and right designation on each pair of nodes with a common parent. A tree isomorphism must preserve this order.

Let $\nu_{2}$ denote the edge of $G$ containing vertices $a_{1}, a_{2}$, and let $T_{2}$ be the tree consisting of the single node $\nu_{2}$. Now let $3 \leq i \leq n$, and let $\nu_{i}, \nu_{i}^{\prime}$ denote the boundary edges of $G_{i}(\mathbf{a})$ immediately to the left and right of $a_{i}$, respectively (this is well defined because $G$ is sequentially convex). Assume that we have already constructed $T_{i-1}$, and that all boundary 


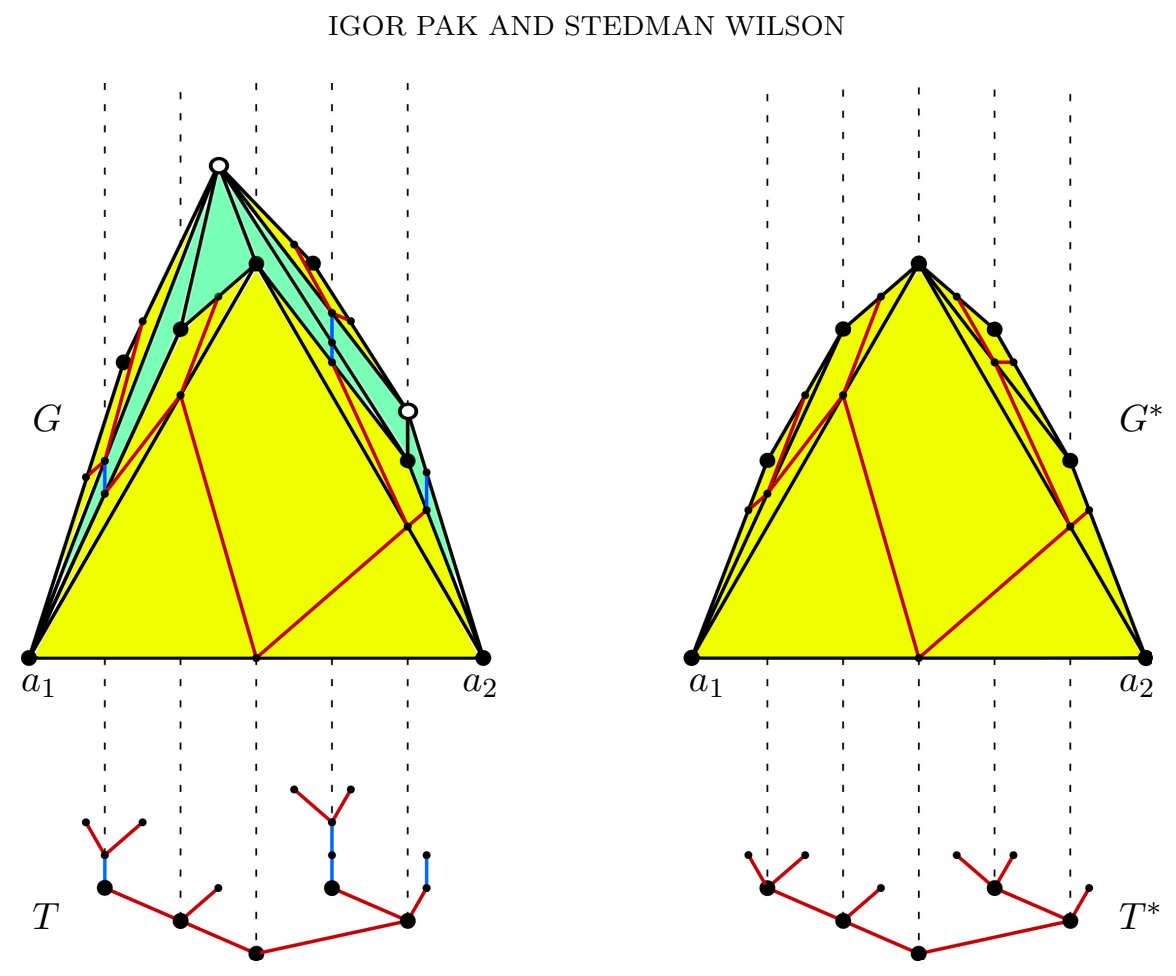

FIgURE 3. The triangulations $G$ and $G^{*}$, together with corresponding trees $T$ and $T^{*}$. Each node of $T$ corresponds to an edge of $G$, and similarly for $T^{*}$ and $G^{*}$. The tree $T^{*}$ is obtained from $T$ by contracting the blue edges. The large nodes of $T$ are the internal nodes of $T^{*}$, and correspond to the vertices of $G^{*}$ other than $a_{1}$ and $a_{2}$.

edges of $G_{i-1}(\mathbf{a})$ are nodes of $T_{i-1}$. Let $\xi, \xi^{\prime}$ be the boundary edges of $G_{i-1}(\mathbf{a})$ such that $\xi$ shares a face with $\nu_{i}$, and $\xi^{\prime}$ shares a face with $\nu_{i}^{\prime}$.

Define $T_{i}=T_{i}(G, \mathbf{a})$ to be the tree obtained from $T_{i-1}$ by adding $\nu_{i}$ and $\nu_{i}^{\prime}$ as nodes, and adding the edges $\left(\xi, \nu_{i}\right)$ and $\left(\xi^{\prime}, \nu_{i}^{\prime}\right)$, designated left and right, respectively. Then clearly all boundary edges of $G_{i}(\mathbf{a})$ are vertices of $T_{i}$. Thus we have a recursively defined sequence of trees $\left(T_{2}, T_{3}, \ldots, T_{n}\right)$, and nodes $\left(\nu_{2}, \nu_{3}, \nu_{3}^{\prime}, \ldots, \nu_{n}, \nu_{n}^{\prime}\right)$, such that $T_{i}$ has nodes $\nu_{2}, \nu_{3}, \nu_{3}^{\prime}, \ldots, \nu_{i}, \nu_{i}^{\prime}$. We call the trees $T_{i}(G, \mathbf{a})$ the shedding trees of $G$, and we write $T=T_{n}$ (see Figure 3$)$. Note that for all $i=2, \ldots, n$, we have $T_{i}(G, \mathbf{a})=T\left(G_{i}(\mathbf{a}),\left(a_{1}, \ldots, a_{i}\right)\right.$ ).

Let $T_{i}^{*}=T_{i}^{*}(G, \mathbf{a})$ be the tree obtained from $T_{i}$ by contracting all edges of the form $(\xi, \sigma)$, where $\sigma=\nu_{j}$ or $\sigma=\nu_{j}^{\prime}$ and $d_{j}\left(a_{j}\right)>2$. These edges are shown in blue in Figure 3 . From the definition of the trees $T_{i}$, it follows that the contracted edges are also exactly the edges of $T_{i}$ containing a node of degree 2 . Therefore each $T_{i}^{*}$ is a full binary tree. We call the trees $T_{i}^{*}$ the reduced trees of $G$, and we write $T^{*}=T_{n}^{*}$. If $d_{i}\left(a_{i}\right)=2$ for all $i \geq 3$, then $T^{*}=T$.

A fundamental idea behind our integer grid embedding is that the reduced tree $T^{*}$ contains all of the critical information needed for carrying out the embedding of $G$. For example, each vertex $a_{i}$ satisfying $d_{i}\left(a_{i}\right)=2$ corresponds to an internal node of $T^{*}$ (shown as large dots in Figure 3). Thus the structure of $T^{*}$ tells us how to horizontally space these vertices, and how to choose the slopes of the boundary edges adjacent to them. On the other hand, when adding vertices $a_{i}$ with $d_{i}\left(a_{i}\right)>2$, our construction will have the property 
that the boundary slopes will be perturbed only slightly, and the horizontal distances between vertices will only increase. Furthermore, throughout our entire construction the total horizontal width of the embedding will remain fixed.

Consider a subsequence of $T_{2}^{*}, T_{3}^{*}, \ldots, T_{n}^{*}$ consisting of the distinct reduced trees of $G$. There is a natural construction which produces a triangulation $G^{*}$ and shedding sequence $\mathbf{a}^{*}$, such that these trees are (isomorphic to) the shedding trees of $G^{*}$. We may view the relationship between $G, T, T^{*}$ and $G^{*}$ as follows. The triangulation $G$, together with a chosen shedding sequence a, determines the shedding tree $T$. From the shedding tree $T$, we may contract edges to obtain the reduced tree $T^{*}$. The structure of $T^{*}$, in turn, tells us how to build a new triangulation $G^{*}$. That is, we may represent this entire process as

$$
G \mapsto T \mapsto T^{*} \mapsto G^{*}
$$

where each arrow indicates a construction that determines the object on the right uniquely from the object on the left. We have already described the first two steps, $G \mapsto T$ and $T \mapsto T^{*}$. The last step, namely the construction of the triangulation $G^{*}$ from $T^{*}$, is the content of the next lemma. This lemma can also be thought of as a special instance of Theorem 3.3, in the case that $d_{i}\left(a_{i}\right)=2$ for all $i \geq 3$. With all three steps in place, we will define $G^{*}$ from $G$ in Section 3.3.

Lemma 3.2 Let $n \geq 3$, and let $\left(t_{2}, \ldots, t_{n}\right)$ be a sequence of full binary trees, such that $t_{i-1}$ is a subtree of $t_{i}$, and $t_{i}$ has $1+2(i-2)$ nodes, for all $i=2, \ldots, n$. Then there is a sequentially convex plane triangulation $H$ with $n$ vertices, and a convex shedding sequence a for $H$, such that $t_{i}$ is isomorphic to $T_{i}(H, \boldsymbol{a})$ for all $i=2, \ldots, n$. Furthermore, $H$ is embedded in a $2(n-2) \times\left(\begin{array}{c}n-1 \\ 2\end{array}\right)$ integer grid, and the boundary edge slopes of $H$ differ by at least 1 .

Proof. Let $m$ and $m^{\prime}$ denote the number of internal nodes of $t_{n}$ to the left and right of the root node, respectively. Without loss of generality we may assume $m \leq m^{\prime}$. Note that $m+m^{\prime}+3=n$. To build the triangulation $H$, we begin by placing $n$ vertices along a convex arc. While any convex arc would suffice, for simplicity of analysis we use the following parabola.

For $-m \leq k \leq m^{\prime}+1$, we define

$$
x_{k}=k, \quad y_{k}=\left(\begin{array}{c}
m^{\prime}+2 \\
2
\end{array}\right)-\left(\begin{array}{c}
|k|+1 \\
2
\end{array}\right) .
$$

Additionally, we define

$$
x_{-m-1}=-x_{m^{\prime}+1}, \quad y_{-m-1}=0 .
$$

Then the $n$ points $\left(x_{k}, y_{k}\right)$ all lie on the (piecewise) parabola defined by

$$
y=-\frac{x^{2}+|x|}{2}+\frac{\left(m^{\prime}+2\right)\left(m^{\prime}+1\right)}{2} .
$$

These points will serve as the vertices of the triangulation $H$ (compare with the vertices of $G^{*}$ in Figure 3).

For all $i=3, \ldots, n$, since $t_{i}$ is full and contains two more nodes than $t_{i-1}$, it follows that $t_{i}$ contains exactly one more internal node than $t_{i-1}$. Let $\left(\xi_{3}, \ldots \xi_{n}\right)$ denote the sequence of internal nodes so obtained. Note that $\xi_{3}$ is the root node of all the trees $t_{i}$, and $t_{2}$ consists of the single node $\xi_{3}$.

As mentioned above, the tree $t_{n}$ is an ordered tree. Consider the linear order on the nodes of $t_{n}$ given by an in-order traversal of $t_{n}$ (in the planar embedding of the tree $T^{*}$ 
shown in Figure 3, this is the same as ordering each node by its $x$-coordinate). This linear order, when restricted to the internal nodes of $t_{n}$, induces a permutation

$$
\omega:\{3, \ldots, n\} \rightarrow\{3, \ldots, n\} .
$$

That is, node $\xi_{i}$ has position $\omega(i)$ in this order. For example, from the definition of $\xi_{3}$ and $m$, we always have $\omega(3)=m+3$. We then define a sequence of points $\mathbf{a}=\left(a_{1}, a_{2}, \ldots, a_{n}\right)$ by

$$
\begin{aligned}
& a_{1}=\left(x_{-m-1}, y_{-m-1}\right), \\
& a_{2}=\left(x_{m^{\prime}+1}, y_{m^{\prime}+1}\right), \\
& a_{i}=\left(x_{\omega(i)-m-3}, y_{\omega(i)-m-3}\right) \quad \text { for } 3 \leq i \leq n .
\end{aligned}
$$

To determine the left and right neighbors of each new vertex $a_{i}$ at each step of the construction of $H$, we define functions $f, g:\{3,4, \ldots, n\} \rightarrow\{1,2, \ldots, n\}$ as follows. If there is an internal node $\xi_{j}$ of the subtree $t_{i}$ that immediately precedes $\xi_{i}$ in the in-order traversal of $t_{i}$, then let $f(i)=j$. Otherwise let $f(i)=1$. Similarly, if there is a node $\xi_{j^{\prime}}$ of $t_{i}$ that immediately succeeds $\xi_{i}$ in the in-order traversal of $t_{i}$, then let $g(i)=j^{\prime}$. Otherwise let $g(i)=2$.

We may now define a sequence of plane triangulations $H_{1}, \ldots, H_{n}$ recursively. Let $H_{1}$ consist of the single vertex $a_{1}$, and let $H_{2}$ consist of the vertices $a_{1}, a_{2}$ and the line segment $a_{1} a_{2}$. Now let $3 \leq i \leq n$, and suppose we have constructed $H_{i-1}$. We obtain $H_{i}$ by adding the vertex $a_{i}$ and the line segments $a_{i} a_{f(i)}$ and $a_{i} a_{g(i)}$ to $H_{i-1}$. This completes the construction of the graphs $H_{2}, \ldots, H_{n}$. We write $H=H_{n}$.

We now check that $\mathbf{a}=\left(a_{1}, \ldots, a_{n}\right)$ is a convex shedding sequence for $H$. By construction, we have immediately that $H_{i}$ is a plane triangulation with $H_{i-1}=H_{i}-\left\{a_{i}\right\}$ for all $i=2, \ldots, n$, and furthermore $d_{i}\left(a_{i}\right)=2$ for all $i \geq 3$. For $k \geq-m$, the slope of the edge between adjacent vertices $\left(x_{k}, y_{k}\right)$ and $\left(x_{k+1}, y_{k+1}\right)$ of $H$ is

$$
\frac{y_{k+1}-y_{k}}{x_{k+1}-x_{k}}=\left(\begin{array}{c}
|k|+1 \\
2
\end{array}\right)-\left(\begin{array}{c}
|k+1|+1 \\
2
\end{array}\right)= \begin{cases}-(k+1) & \text { if } k \geq 0, \\
-k & \text { if } k<0 .\end{cases}
$$

Additionally, the slope of the edge between $\left(x_{-m-1}, y_{-m-1}\right)$ and $\left(x_{-m}, y_{-m}\right)$ is

$$
\begin{aligned}
& \frac{y_{-m}-y_{-m-1}}{x_{-m}-x_{-m-1}}=\frac{y_{-m}}{-m+\left(m^{\prime}+1\right)}=\frac{1}{m^{\prime}-m+1}\left[\left(\begin{array}{c}
m^{\prime}+2 \\
2
\end{array}\right)-\left(\begin{array}{c}
m+1 \\
2
\end{array}\right)\right] \\
& =\frac{\left(m^{\prime}+2\right)\left(m^{\prime}+1\right)-(m+1) m}{2\left(m^{\prime}-m+1\right)}=\frac{\left(m^{\prime}-m+1\right)\left(m^{\prime}+m+2\right)}{2\left(m^{\prime}-m+1\right)} \\
& =\frac{m^{\prime}+m+2}{2} \geq \frac{m+m+2}{2}=m+1 .
\end{aligned}
$$

Thus the boundary edge slopes of $H$ are strictly decreasing from left to right, and differ by at least 1 . Since $d_{i}\left(a_{i}\right)=2$ for all $i \geq 3$, the same is true for the boundary edge slopes of each $H_{i}$. It follows that $\mathbf{F}\left(H_{i}\right)$ is projectively convex, for all $i \geq 3$. Hence $\mathbf{a}$ is a convex shedding sequence for $H$.

To see that $t_{i}$ is isomorphic to $T_{i}(H, \mathbf{a})$ for all $i=2, \ldots, n$, we construct an explicit isomorphism. We define a map $\psi_{2}: t_{2} \rightarrow T_{2}(H, \mathbf{a})$ by $\psi_{2}\left(\xi_{3}\right)=a_{f(3)} a_{g(3)}=a_{1} a_{2}$, which is trivially an isomorphism. For $i \geq 3$, and $j=3, \ldots, i$, let $\xi_{j}^{-}$and $\xi_{j}^{+}$denote the left and right child, respectively, of the internal node $\xi_{j}$ of $t_{i}$. We define a map $\psi_{i}: t_{i} \rightarrow T_{i}(H, \mathbf{a})$ by

$$
\begin{aligned}
\psi_{i}\left(\xi_{j}\right) & =a_{f(j)} a_{g(j)}, \\
\psi_{i}\left(\xi_{j}^{-}\right) & =a_{j} a_{f(j)}, \\
\psi_{i}\left(\xi_{j}^{+}\right) & =a_{j} a_{g(j)} \quad \text { for } j=3, \ldots, i .
\end{aligned}
$$


From the definition of the functions $f$ and $g$, it follows that $\psi$ is well-defined and bijective. Since the triangle $a_{j} a_{f(j)} a_{g(j)}$ is a face of $H_{j}$ for all $j=3, \ldots, i$, the pairs $\left(a_{f(j)} a_{g(j)}, a_{j} a_{f(j)}\right)$ and $\left(a_{f(j)} a_{g(j)}, a_{j} a_{g(j)}\right)$ are edges of $T_{i}(H, \mathbf{a})$. Thus $\psi_{i}$ is a tree isomorphism. We may think of $\psi_{n}$ as providing a correspondence between the internal node $\xi_{i}$ and the vertex $a_{i}$ (whose neighbors in $H_{i}$ are $a_{f(i)}$ and $\left.a_{g(i)}\right)$, for all $i=3, \ldots, n$ (see Figure 3 ).

Finally, the width of the grid is

$$
x_{m^{\prime}+1}-x_{-m-1}=2 x_{m^{\prime}+1}=2\left(m^{\prime}+1\right) \leq 2(n-2),
$$

and the height of the grid is

$$
y_{0}=\left(\begin{array}{c}
m^{\prime}+2 \\
2
\end{array}\right) \leq\left(\begin{array}{c}
n-1 \\
2
\end{array}\right) .
$$

Therefore $H$ is embedded in an integer grid of size $2(n-2) \times\left(\begin{array}{c}n-1 \\ 2\end{array}\right)$.

3.3. The Integer Grid Embedding. Consider a plane triangulation $G$ with $n$ vertices and shedding sequence a, with reduced trees $T_{i}^{*}=T_{i}^{*}(G, \mathbf{a})$. For each $i \in\{2,3, \ldots, n\}$ let $\rho(i)$ denote the least integer for which $T_{\rho(i)}^{*}=T_{i}^{*}$, and let $\rho(1)=1$. Clearly $\rho(2)=2$ and $\rho(3)=3$ as well. For the tree $T$ in Figure 3, we have $\rho(i)=1,2,3,4,5,5,5,6,7$ for $i=1,2, \ldots 9$.

The sequence of distinct trees $T_{1}^{*}, T_{2}^{*}, \ldots, T_{\rho(n)}^{*}$ satisfies the hypotheses of Lemma 3.2. Therefore we let $G^{*}$ denote the sequentially convex triangulation constructed exactly as in Lemma 3.2 from this sequence of trees. That is, $G^{*}$ is the triangulation $H$ in the notation of the lemma, and $G^{*}$ has the exact vertex coordinates given in the lemma. We let $\mathbf{a}^{*}=\left(a_{1}^{*}, \ldots, a_{\rho(n)}^{*}\right)$ denote the corresponding convex shedding sequence of $G^{*}$ produced by Lemma 3.2. For brevity we will write $G_{i}^{*}=G_{i}^{*}\left(\mathbf{a}^{*}\right)$ for all $i=1,2, \ldots, \rho(n)$, so in particular $G_{\rho(n)}^{*}=G^{*}$. We call the $G_{i}^{*}$ the reduced triangulations of $G$ (see Figure 3 ). Note that each vertex $a_{i}^{*}$ has degree 2 in $G_{i}^{*}$. So we may think of $G^{*}$ as being obtained from $G$ by "throwing away" all vertices $a_{i}$ for which $d_{i}\left(a_{i}\right)>2$. It was this property that originally motivated our definition of $G^{*}$.

As we will see, the triangulation $G^{*}$ will tell us exactly how to add vertices of degree 2 , in our construction of a sequentially convex embedding of $G$. A particular property of the reduced triangulations makes this possible. Namely, for any boundary edge $e$ of $G_{i}$, there is a corresponding boundary edge $e^{*}$ of $G_{\rho(i)}^{*}$, which we define as follows. The edge $e$ is a node of the shedding tree $T_{i}(G, \mathbf{a})$. As described above, we obtain $T_{i}^{*}$ from $T_{i}$ by contracting all edges of $T_{i}$ containing a node of degree 2 . This contraction identifies the node $e$ with a unique node of $T_{i}^{*}=T_{\rho(i)}^{*}$ which, on constructing $G^{*}$ from $T^{*}$ as in Theorem 3.2, corresponds to a unique edge $e^{*}$ of $G_{\rho(i)}^{*}$.

Theorem 3.3 Let $G$ be a plane triangulation with $n$ vertices and boundary edge uv, and let $\boldsymbol{a}=\left(a_{1}, \ldots, a_{n}\right)$ be a shedding sequence for $G$ with $u=a_{1}, v=a_{2}$. Then $G$ has $a$ geometric embedding $G^{\prime}$ in $a 4 n^{3} \times 8 n^{5}$ integer grid, such that the corresponding sequence $\boldsymbol{a}^{\prime}=\left(a_{1}^{\prime}, \ldots, a_{n}^{\prime}\right)$ is a convex shedding sequence for $G^{\prime}$.

Proof. We recursively construct a sequence of graphs $G_{1}^{\prime}, \ldots, G_{n}^{\prime}$, and a sequence of vertices $a_{1}^{\prime}, \ldots, a_{n}^{\prime}$, such that each $G_{i}^{\prime}$ is a geometric embedding of $G_{i}$ with convex shedding sequence $\mathbf{a}^{\prime}=\left(a_{1}^{\prime}, \ldots, a_{i}^{\prime}\right)$, where $a_{i}^{\prime}$ is the vertex of $G^{\prime}$ corresponding to $a_{i}$. Let $G_{i}^{*}$ denote the reduced triangulations of $G$, and let $\mathbf{a}^{*}=\left(a_{1}^{*}, \ldots, a_{R}^{*}\right)$ denote the corresponding shedding sequence for $G^{*}$. Let $m^{\prime}$ denote the number of vertices of $G^{*}$ lying between $a_{3}^{*}$ and $a_{2}^{*}$, and $m$ the 


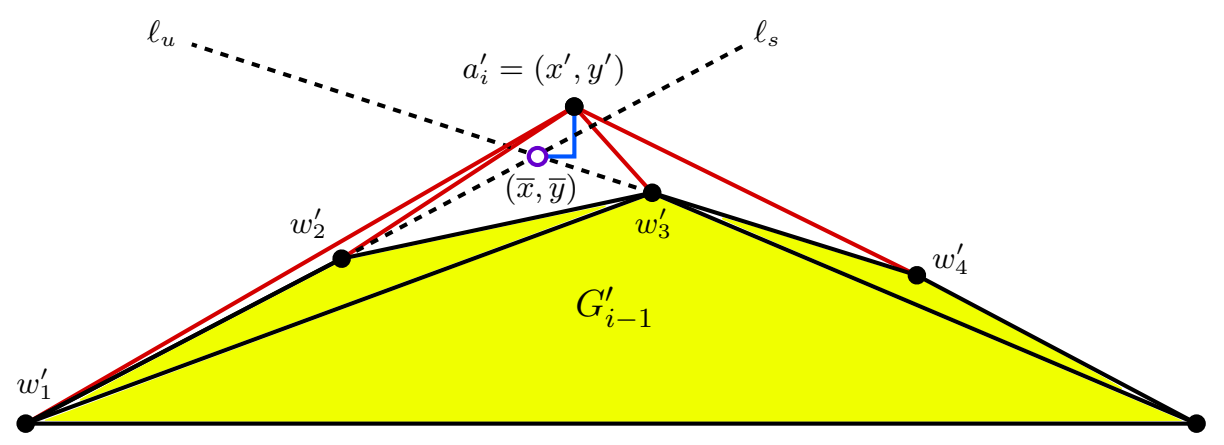

FiguRE 4. The construction of vertex $a_{i}^{\prime}$ when $d_{i}\left(a_{i}\right)>2$. In this example, $d_{i}\left(a_{i}\right)=4$.

number of vertices lying between $a_{1}^{*}$ and $a_{3}^{*}$. Then $m^{\prime}+m+3=n$. Without loss of generality we may assume that $m \leq m^{\prime}$.

We first scale $G^{*}$ to obtain a much larger triangulation, which we will use to construct the triangulations $G_{i}^{\prime}$. Specifically, let $\alpha=2 n^{2}+n+1$ and $\beta=2 n \alpha$. These are the smallest scaling factors that ensure that we have "enough room" to carry out our constructions. In particular, $\alpha$ will ensure that we have enough horizontal room to add new points, and $\beta$ will ensure that we have enough vertical room to maintain convexity at each step of the construction.

We define $Z_{i}$ to be the result of scaling $G_{i}^{*}$ by a factor of $\alpha$ in the $x$ dimension and $\beta$ in the $y$ dimension. That is, for each $i=1, \ldots, R$, we define $z_{i}=\left(\alpha x\left(a_{i}^{*}\right), \beta y\left(a_{i}^{*}\right)\right)$. Then $\mathbf{z}=\left(z_{1}, \ldots, z_{R}\right)$ is the shedding sequence for $Z_{R}$ corresponding to $\mathbf{a}^{*}$. We write $Z=Z_{R}$.

Define $a_{1}^{\prime}=z_{1}$ and $a_{2}^{\prime}=z_{2}$. Take $G_{1}^{\prime}$ to consist of the single vertex $a_{1}^{\prime}$, and take $G_{2}^{\prime}$ to consist of the vertices $a_{1}^{\prime}, a_{2}^{\prime}$, together with the line segment $a_{1}^{\prime} a_{2}^{\prime}$. Now let $3 \leq i \leq n$, and suppose we have constructed $G_{i-1}^{\prime}$. To define $a_{i}^{\prime}$, we consider two cases, namely whether $d_{i}\left(a_{i}\right)=2$ or $d_{i}\left(a_{i}\right)>2$.

Construction of $a_{i}^{\prime}$, in the case $d_{i}\left(a_{i}\right)>2$. If $d_{i}\left(a_{i}\right)>2$, then let $w_{1}, \ldots, w_{k}$ denote the neighbors of $a_{i}$ in $G_{i}$, and let $w_{1}^{\prime}, \ldots, w_{k}^{\prime}$ denote the corresponding vertices of $G_{i-1}^{\prime}$, ordered from left to right. Let $s$ denote the slope of the edge $w_{1}^{\prime} w_{2}^{\prime}$, and $u$ the slope of the edge $w_{k-1}^{\prime} w_{k}^{\prime}$. Let $\ell_{s}$ denote the line of slope $s$ containing the point $w_{1}^{\prime}$, and $\ell_{u}$ the line of slope $u$ containing the point $w_{k}^{\prime}$. We denote by $(\bar{x}, \bar{y})$ the point of intersection of the lines $\ell_{s}$ and $\ell_{u}$. We then move $(\bar{x}, \bar{y})$ to the integer grid point $\left(x^{\prime}, y^{\prime}\right)$ defined as follows. Let $x^{\prime}=\lceil\bar{x}\rceil$ and $\gamma=x^{\prime}-\bar{x}$, and let $y^{\prime}=\lceil\bar{y}\rceil+\lfloor\gamma s\rfloor+1$. We now define $a_{i}^{\prime}=\left(x^{\prime}, y^{\prime}\right)$ (see Figure 4). We obtain $G_{i}^{\prime}$ from $G_{i-1}^{\prime}$ by adding the vertex $a_{i}^{\prime}$, together with all line segments between $a_{i}^{\prime}$ and the vertices $w_{1}^{\prime}, \ldots, w_{k}^{\prime}$.

Construction of $a_{i}^{\prime}$, in the case $d_{i}\left(a_{i}\right)=2$. If $d_{i}\left(a_{i}\right)=2$, then let $\Delta$ be the triangle of $Z_{\rho(i)}$ containing $z_{\rho(i)}$. Let $w_{1}, w_{2}$ denote the boundary neighbors of $a_{i}$ in $G_{i}$, and let $w_{1}^{\prime}, w_{2}^{\prime}$ denote the corresponding vertices of $G_{i-1}^{\prime}$, so that $w_{1}^{\prime}$ lies to the left of $w_{2}^{\prime}$. We are going to construct a triangle $\Delta^{\prime}$, such that $\Delta^{\prime}$ is the image of $\Delta$ under an affine map which is the composition of a uniform scaling and a translation. Furthermore, we will place $\Delta^{\prime}$ in a specific position with respect to the triangulation $G_{i-1}^{\prime}$. In particular, if $v_{1}, v_{2}, v_{3}$ denote the vertices of $\Delta^{\prime}$, we require that $x\left(v_{1}\right)=x\left(w_{1}^{\prime}\right), v_{2}=w_{2}^{\prime}$, and $x\left(v_{1}\right)<x\left(v_{3}\right)<x\left(v_{2}\right)$ (see Figure 5). It is easily verified that these conditions, together with the requirement that $\Delta^{\prime}$ is a scaled, translated copy of $\Delta$, determine the vertices $v_{1}, v_{2}, v_{3}$ of $\Delta^{\prime}$ uniquely. 

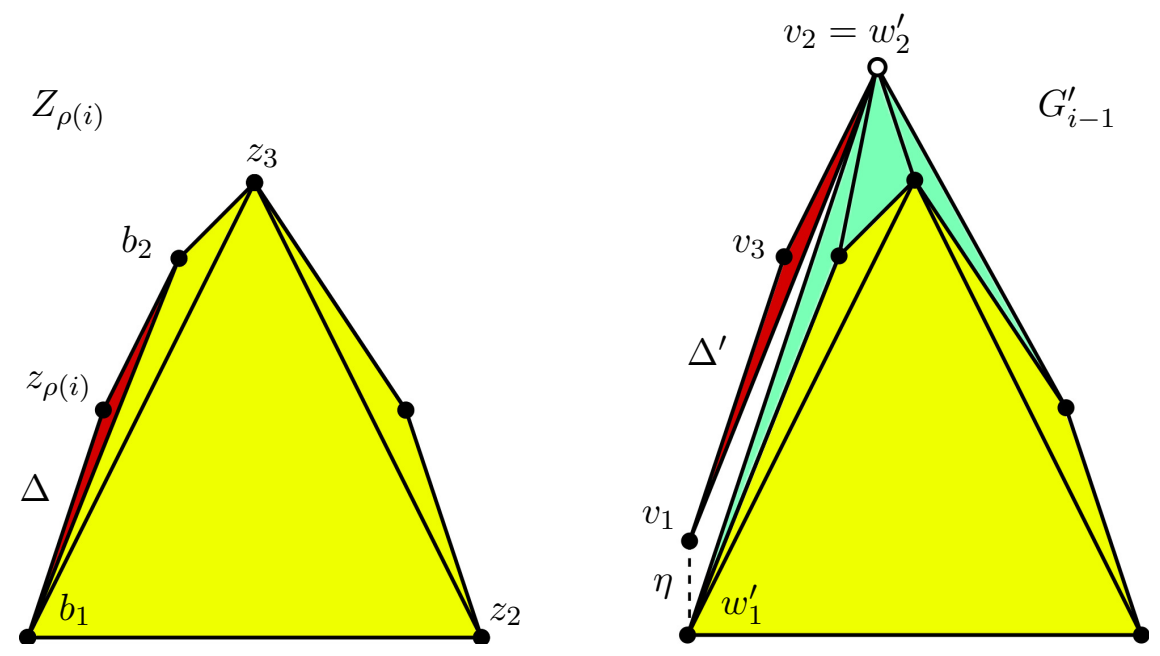

FiguRE 5. The first stage of the construction of vertex $a_{i}^{\prime}$ when $d_{i}\left(a_{i}\right)=2$.

The red triangles $\Delta$ and $\Delta^{\prime}$ differ by a uniform scaling and a translation.

To define the new vertex $a_{i}^{\prime}$, we start by applying a vertical shearing to the triangle $\Delta^{\prime}$, namely the unique shearing that fixes $v_{2}=w_{2}^{\prime}$ and maps $v_{1}$ to $w_{1}^{\prime}$. We will denote the image of $v_{3}$ under this shearing by $\overline{v_{3}}$. So in terms of the vertices of $Z_{\rho(i)}$ and $G_{i-1}^{\prime}$, the point $\overline{v_{3}}$ is defined as follows.

Let $\eta=y\left(v_{1}\right)-y\left(w_{1}^{\prime}\right)$. Let $b_{1}$ and $b_{2}$ denote the left and right boundary neighbors, respectively, of $z_{\rho(i)}$ in $Z_{\rho(i)}$. By taking the ratio

$$
\kappa=\frac{x\left(b_{2}\right)-x\left(z_{\rho(i)}\right)}{x\left(b_{2}\right)-x\left(b_{1}\right)},
$$

we may now define $\overline{v_{3}}=\left(x\left(v_{3}\right), y\left(v_{3}\right)-\kappa \eta\right)$.

We move $\overline{v_{3}}$ to the integer grid point

$$
v_{3}^{\prime}= \begin{cases}\left(\left\lfloor x\left(\overline{v_{3}}\right)\right\rfloor,\left\lceil y\left(\overline{v_{3}}\right)\right\rceil\right) & \text { if } x\left(z_{\rho(i)}\right) \leq 0, \\ \left(\left\lceil x\left(\overline{v_{3}}\right)\right\rceil,\left\lceil y\left(\overline{v_{3}}\right)\right\rceil\right) & \text { if } x\left(z_{\rho(i)}\right)>0,\end{cases}
$$

and define $a_{i}^{\prime}=v_{3}^{\prime}$. We obtain $G_{i}^{\prime}$ from $G_{i-1}^{\prime}$ by adding the vertex $a_{i}^{\prime}$ and the two line segments between $a_{i}^{\prime}$ and the vertices $w_{1}^{\prime}, w_{2}^{\prime}$.

\section{Verification of the construction.}

We have now explicitly described the construction of $G^{\prime}$, from which it is clear that $G_{i}^{\prime} \sim G_{i}$ for all $i=1, \ldots, n$. It remains to show that the above constructions actually produce a convex shedding sequence $\mathbf{a}^{\prime}=\left(a_{1}^{\prime}, \ldots, a_{n}^{\prime}\right)$ for $G^{\prime}$, and that $G^{\prime}$ lies in the grid size indicated.

Since $G_{i}^{\prime} \sim G_{i}$, the edges of $G_{i}^{\prime}$ and $G_{i}$ are in correspondence. Thus every boundary edge $e$ of $G_{i}^{\prime}$ has a corresponding boundary edge $e^{*}$ of $G_{\rho(i)}^{*}$, as defined above. We then write $Z(e)$ for the edge of $Z_{\rho(i)}$ corresponding to $e^{*}$. Note that if $e^{*}$ has slope $s$, then $Z(e)$ has slope $\frac{\beta}{\alpha} s$. In particular, since $m^{\prime}+1$ is the largest magnitude of the slope of any edge of $G^{*}$, we see that $\frac{\beta}{\alpha}\left(m^{\prime}+1\right)=2 n\left(m^{\prime}+1\right)$ is the largest magnitude of the slope of any edge of $Z$. Let $M$ denote this slope, and note that $M \leq 2 n^{2}$. Note also that the absolute difference of two boundary edge slopes of $Z$ is at least $\frac{\beta}{\alpha}=2 n$. It follows immediately that for each $i=1, \ldots, R$, the absolute difference of two boundary edge slopes of $Z_{i}$ is at least $2 n$. 
Clearly, the horizontal width of the grid remains constant throughout the construction. Specifically, the triangulations $G_{1}^{\prime}, G_{2}^{\prime}, \ldots, G_{n}^{\prime}$ all have the same width $\alpha 2(n-2)$, which is the width of the $Z_{i}$. So to show that the construction is sequentially convex, and that the bound on the height of $G^{\prime}$ is correct, we will calculate how the boundary slopes are modified when we add the new vertex $a_{i}^{\prime}$ in the two cases $d_{i}\left(a_{i}\right)=2$ and $d_{i}\left(a_{i}\right)>2$.

For points $v_{1}, v_{2} \in \mathbb{R}^{2}$ and $e=v_{1} v_{2}$ the line segment between them, we write

$$
x(e)=\left|x\left(v_{1}\right)-x\left(v_{2}\right)\right| \quad \text { and } \quad y(e)=\left|y\left(v_{1}\right)-y\left(v_{2}\right)\right| .
$$

For each $3 \leq i \leq n$, let $\mathcal{P}(i)$ denote the conjunction of the following three properties, that we wish to show:

$\mathcal{P}(i, 1)$. For every boundary edge $e$ of $G_{i}^{\prime}$, we have $x(e) \geq x(Z(e))$.

$\mathcal{P}(i, 2)$. For every boundary edge $e$ of $G_{i}^{\prime}$, the slopes of $e$ and $Z(e)$ differ by at most $i$.

$\mathcal{P}(i, 3) . \quad G_{i}^{\prime} \sim G_{i}$ and $G_{i}^{\prime}$ has convex shedding sequence $\left(a_{1}^{\prime}, \ldots a_{i}^{\prime}\right)$.

To prove $\mathcal{P}(i)$ for each $i=3, \ldots, n$, we proceed by induction on $i$.

For $i=3$, note that $a_{1}^{\prime}=z_{1}, a_{2}^{\prime}=z_{2}$, and the vertex $a_{3}^{\prime}$ is chosen so that in particular the triangle $\left(a_{1}^{\prime} a_{2}^{\prime} a_{3}^{\prime}\right)$ is a scaling of the triangle $\left(z_{1} z_{2} z_{3}\right)=\mathbf{F}\left(Z_{3}\right)$. This implies that $a_{3}^{\prime}=z_{3}$. Thus $G_{3}^{\prime}=Z_{3}$, which immediately establishes $\mathcal{P}(3)$.

Now let $i>3$, and suppose that $\mathcal{P}(i-1)$ holds. As in the construction, we consider separately the cases $d_{i}\left(a_{i}\right)>2$ and $d_{i}\left(a_{i}\right)=2$.

Verification of $\mathcal{P}(i)$ in the case $d_{i}\left(a_{i}\right)>2$. From the definition of $Z(e)$, we have $Z\left(w_{1}^{\prime} a_{i}^{\prime}\right)=Z\left(w_{1}^{\prime} w_{2}^{\prime}\right)$ and $Z\left(a_{i}^{\prime} w_{k}^{\prime}\right)=Z\left(w_{k-1}^{\prime} w_{k}^{\prime}\right)$. Note that $x\left(w_{2}^{\prime}\right) \leq \bar{x} \leq x\left(w_{k-1}^{\prime}\right)$, and thus $x\left(w_{2}^{\prime}\right) \leq x\left(a_{i}^{\prime}\right) \leq x\left(w_{k-1}^{\prime}\right)$, because $x\left(a_{i}^{\prime}\right)=\lceil\bar{x}\rceil$ and $x\left(w_{2}^{\prime}\right)$ and $x\left(w_{k-1}^{\prime}\right)$ are integers. Therefore

$$
x\left(w_{1}^{\prime} a_{i}^{\prime}\right)=x\left(a_{i}^{\prime}\right)-x\left(w_{1}^{\prime}\right) \geq x\left(w_{2}^{\prime}\right)-x\left(w_{1}^{\prime}\right)=x\left(w_{1}^{\prime} w_{2}^{\prime}\right) \geq x\left(Z\left(w_{1}^{\prime} w_{2}^{\prime}\right)\right)=x\left(Z\left(w_{1}^{\prime} a_{i}^{\prime}\right)\right),
$$

where the last inequality follows from $\mathcal{P}(i-1,1)$. Similarly, we have $x\left(a_{i}^{\prime} w_{k}^{\prime}\right) \geq x\left(Z\left(a_{i}^{\prime} w_{k}^{\prime}\right)\right)$. Thus $\mathcal{P}(i, 1)$ holds.

We now show that the slopes of the boundary edges of $G_{i}^{\prime}$ containing $a_{i}^{\prime}$ differ only slightly from the slopes $s$ and $u$ defined in the above construction. That is, let $s^{\prime}$ denote the slope of the line passing through $w_{1}^{\prime}$ and $a_{i}^{\prime}=\left(x^{\prime}, y^{\prime}\right)$, and let $u^{\prime}$ denote the slope of the line passing through $a_{i}^{\prime}$ and $w_{k}^{\prime}$. First note that

$$
y^{\prime}-\bar{y}=(\lceil\bar{y}\rceil-\bar{y})+\lfloor\gamma s\rfloor+1 \geq\lfloor\gamma s\rfloor+1>\gamma s .
$$

Therefore

$$
s^{\prime}-s=\frac{y^{\prime}-y\left(w_{1}^{\prime}\right)}{\bar{x}+\gamma-x\left(w_{1}^{\prime}\right)}-s=\frac{y^{\prime}-y\left(w_{1}^{\prime}\right)-\left(\bar{x}-x\left(w_{1}^{\prime}\right)\right) s-\gamma s}{\bar{x}+\gamma-x\left(w_{1}^{\prime}\right)}=\frac{\left(y^{\prime}-\bar{y}\right)-\gamma s}{\bar{x}+\gamma-x\left(w_{1}^{\prime}\right)}>0 .
$$

On the other hand,

$$
\begin{aligned}
& s^{\prime}-s=\frac{\left(y^{\prime}-\bar{y}\right)-\gamma s}{\bar{x}+\gamma-x\left(w_{1}^{\prime}\right)}=\frac{(\lceil\bar{y}\rceil-\bar{y})+(\lfloor\gamma s\rfloor-\gamma s)+1}{\bar{x}+\gamma-x\left(w_{1}^{\prime}\right)} \\
& <\frac{2}{\bar{x}+\gamma-x\left(w_{1}^{\prime}\right)} \leq \frac{2}{\bar{x}-x\left(w_{1}^{\prime}\right)} \leq \frac{2}{x\left(w_{2}^{\prime}\right)-x\left(w_{1}^{\prime}\right)} \\
& \leq \frac{2}{x\left(Z\left(w_{2}^{\prime} w_{1}^{\prime}\right)\right)} \leq \frac{2}{\alpha} \leq 1
\end{aligned}
$$

In the last line we have used $\mathcal{P}(i-1,1)$. 
Since $s>u$, we have $y^{\prime}-\bar{y}>\gamma s>\gamma u$. From this, together with the fact that $x^{\prime} \geq \bar{x}$, it follows that $u-u^{\prime}>0$. By $\mathcal{P}(i-1,2)$, we have $|s| \leq M+(i-1)$. Thus

$$
\begin{aligned}
& u-u^{\prime}=\frac{y\left(w_{k}^{\prime}\right)-\bar{y}}{x\left(w_{k}^{\prime}\right)-\bar{x}}-\frac{y\left(w_{k}^{\prime}\right)-y^{\prime}}{x\left(w_{k}^{\prime}\right)-x^{\prime}} \leq \frac{y\left(w_{k}^{\prime}\right)-\bar{y}}{x\left(w_{k}^{\prime}\right)-x^{\prime}}-\frac{y\left(w_{k}^{\prime}\right)-y^{\prime}}{x\left(w_{k}^{\prime}\right)-x^{\prime}}=\frac{y^{\prime}-\bar{y}}{x\left(w_{k}^{\prime}\right)-x^{\prime}} \\
& =\frac{(\lceil\bar{y}\rceil-\bar{y})+\lfloor\gamma\rfloor+1}{x\left(w_{k}^{\prime}\right)-x^{\prime}}<\frac{|s|+2}{x\left(w_{k}^{\prime}\right)-x^{\prime}} \leq \frac{|s|+2}{x\left(w_{k}^{\prime}\right)-x\left(w_{k-1}^{\prime}\right)} \leq \frac{|s|+2}{x\left(Z\left(w_{k-1}^{\prime} w_{k}^{\prime}\right)\right)} \\
& \leq \frac{|s|+2}{\alpha} \leq \frac{M+(i-1)+2}{\alpha} \leq \frac{2 n^{2}+i+1}{\alpha} \leq \frac{2 n^{2}+n+1}{\alpha}=1 .
\end{aligned}
$$

In the second line we have used $\mathcal{P}(i-1,1)$.

Let $s_{Z}$ denote the slope of the edge $Z\left(w_{1}^{\prime} w_{2}^{\prime}\right)$, and let $u_{Z}$ denote the slope of $Z\left(w_{k-1}^{\prime} w_{k}^{\prime}\right)$. By $\mathcal{P}(i-1,2)$, we have $\left|s-\left({ }_{Z} s\right)\right| \leq i-1$ and $\left|u-u_{Z}\right| \leq i-1$. Thus

$$
\left|s^{\prime}-s_{Z}\right| \leq\left|s^{\prime}-s\right|+\left|s-s_{Z}\right| \leq 1+(i-1)=i,
$$

and similarly $\left|u^{\prime}-u_{Z}\right| \leq i$, so $\mathcal{P}(i, 2)$ holds.

Since $s^{\prime}-s>0$ and $u-u^{\prime}>0$, each line segment $a_{i}^{\prime} w_{j}^{\prime}$ intersects $G_{i-1}^{\prime}$ only in the vertex $w_{j}^{\prime}$, for all $j=1, \ldots k$. Thus $G_{i}^{\prime}$ is a plane triangulation, and $G_{i}^{\prime} \sim G_{i}$. It remains to show that $\mathbf{F}\left(G_{i}^{\prime}\right)$ is projectively convex, in order to establish $\mathcal{P}(i, 3)$. To do this, we will show that when the slope $s$ is changed to $s^{\prime}$ for example, convexity is preserved at the vertex $w_{1}^{\prime}$. That is, the slope $s^{\prime}$, while greater than $s$, is still less than the slope of the boundary edge to the left of $w_{1}^{\prime} w_{2}^{\prime}$.

Let $\hat{s}$ denote the slope of the boundary edge of $G_{i}^{\prime}$ adjacent and to the left of $w_{1}^{\prime}$, if such an edge exists, and let $\hat{u}$ denote the slope of the boundary edge of $G_{i}^{\prime}$ adjacent and to the right of $w_{k}^{\prime}$, if such an edge exists. Let $\hat{s}_{Z}$ and $\hat{u}_{Z}$ denote the boundary slopes of $Z_{h(i-1)}$ corresponding to $\hat{s}$ and $\hat{u}$, respectively. By $\mathcal{P}(i-1,2)$, we have $s-s_{Z} \leq i-1$ and $\hat{s}_{Z}-\hat{s} \leq i-1$. Therefore

$$
\begin{gathered}
\hat{s}-s^{\prime}=\left(\hat{s}_{Z}-s_{Z}\right)-\left(\hat{s}_{Z}-\hat{s}\right)-\left(s-s_{Z}\right)-\left(s^{\prime}-s\right) \\
\geq 2 n-(i-1)-(i-1)-1=2 n-2 i+1>0 .
\end{gathered}
$$

An analogous calculation shows that $u^{\prime}-\hat{u}>0$. Thus $w_{1}^{\prime}$ and $w_{k}^{\prime}$ are convex vertices of $G_{i}^{\prime}$. Because the region $\mathbf{F}\left(G_{i-1}^{\prime}\right)$ is projectively convex by $\mathcal{P}(i-1,3)$, we conclude that $\mathbf{F}\left(G_{i}^{\prime}\right)$ is projectively convex. The sequence $\left(a_{1}^{\prime}, \ldots, a_{i-1}^{\prime}\right)$ is a convex shedding sequence for $G_{i-1}^{\prime}$ by $\mathcal{P}(i-1,3)$, hence $\left(a_{1}^{\prime}, \ldots, a_{i}^{\prime}\right)$ is a convex shedding sequence for $G_{i}^{\prime}$. Thus $\mathcal{P}(i, 3)$ holds. We have now established $\mathcal{P}(i)$ in the case that $d_{i}\left(a_{i}\right)>2$.

Verification of $\mathcal{P}(i)$ in the case $d_{i}\left(a_{i}\right)=2$. First note that by $\mathcal{P}(i-1,1)$, we have

$$
x\left(v_{1} v_{2}\right)=x\left(w_{1}^{\prime} w_{2}^{\prime}\right) \geq x\left(Z\left(w_{1}^{\prime} w_{2}^{\prime}\right)\right)=x\left(b_{1} b_{2}\right) .
$$

This implies that $x\left(w_{1}^{\prime} \overline{v_{3}}\right)=x\left(v_{1} v_{3}\right) \geq x\left(b_{1} z_{\rho(i)}\right)$, because $\Delta^{\prime}$ is a scaled, translated copy of $\Delta$. Since $x\left(a_{i}^{\prime}\right)$ is either $\left\lfloor x\left(\overline{v_{3}}\right)\right\rfloor$ or $\left\lceil x\left(\overline{v_{3}}\right)\right\rceil$, and $x\left(b_{1} z_{\rho(i)}\right)$ and $x\left(w_{1}^{\prime}\right)$ are integers, we also have

$$
x\left(w_{1}^{\prime} a_{i}^{\prime}\right) \geq x\left(b_{1} z_{\rho(i)}\right)=x\left(Z\left(w_{1}^{\prime} a_{i}^{\prime}\right)\right) .
$$

Similarly, we obtain $x\left(a_{i}^{\prime} w_{2}^{\prime}\right) \geq x\left(z_{\rho(i)} b_{2}\right)=x\left(Z\left(a_{i}^{\prime} w_{2}^{\prime}\right)\right)$. Thus $\mathcal{P}(i, 1)$ holds.

To establish $\mathcal{P}(i, 2)$, we first consider an important pair of corresponding slopes in the construction. Let $r$ denote the slope of the edge $w_{1}^{\prime} w_{2}^{\prime}$ of $G_{i-1}^{\prime}$, and let $Z(r)$ denote the slope of the corresponding edge $Z\left(w_{1}^{\prime} w_{2}^{\prime}\right)=b_{1} b_{2}$ of $Z_{\rho(i-1)}$. Since the triangle $\Delta^{\prime}$ is a scaled, translated copy of $\Delta$, we see that $Z(r)$ is also the slope of the edge $v_{1} v_{2}$ of $\Delta^{\prime}$. Let $\varepsilon=r-Z(r)$. 
We now consider the slopes of the other two edges of $\Delta^{\prime}$. Namely, let $q_{1}$ and $q_{2}$ denote the slopes of the line segments $v_{1} v_{3}$ and $v_{3} v_{2}$, respectively. Since $\Delta^{\prime}$ is a scaled, translated copy of $\Delta$, these slopes $q_{1}$ and $q_{2}$ are also the slopes of the boundary edges $b_{1} z_{\rho(i)}$ and $z_{\rho(i)} b_{2}$ of $Z_{\rho(i)}$, respectively. Let $\overline{q_{1}}$ and $\overline{q_{2}}$ denote the slopes of the line segments $\overline{v_{3}} w_{1}^{\prime}$ and $\overline{v_{3}} w_{2}^{\prime}$, respectively. Then the vertical shearing of $\Delta^{\prime}$, described above, takes lines of slopes $q_{1}, q_{2}$, and $Z(r)$ to lines of slopes $\overline{q_{1}}, \overline{q_{2}}$, and $r$, respectively. Since a vertical shearing adds the same constant to the slope of every line, we conclude that

$$
\overline{q_{1}}-q_{1}=\overline{q_{2}}-q_{2}=r-Z(r)=\varepsilon .
$$

We now investigate how the slopes $\overline{q_{1}}$ and $\overline{q_{2}}$ change when we move $\overline{v_{3}}$ to the integer point $v_{3}^{\prime}=a_{i}^{\prime}$. We may assume without loss of generality that $x\left(z_{\rho(i)}\right)<0$, and hence that $a_{i}^{\prime}=\left(\left\lfloor x\left(\overline{v_{3}}\right)\right\rfloor,\left\lceil y\left(\overline{v_{3}}\right)\right\rceil\right)$, as the other case is treated identically. We will let $q_{1}^{\prime}$ and $q_{2}^{\prime}$ denote the slopes that result from replacing $\overline{v_{3}}$ with $v_{3}^{\prime}=a_{i}^{\prime}$. That is, let $q_{1}^{\prime}$ be the slope of the line passing through $a_{i}^{\prime}$ and $w_{1}^{\prime}$, and let $q_{2}^{\prime}$ be the slope of the line passing through $a_{i}^{\prime}$ and $w_{2}^{\prime}$.

By $\mathcal{P}(i-1,2)$, we have $|\varepsilon| \leq i-1$. Therefore we obtain

$$
\begin{aligned}
& q_{1}^{\prime}-\overline{q_{1}}=\frac{\left\lceil y\left(\overline{v_{3}}\right)\right\rceil-y\left(w_{1}^{\prime}\right)}{\left\lfloor x\left(\overline{v_{3}}\right)\right\rfloor-x\left(w_{1}^{\prime}\right)}-\overline{q_{1}}<\frac{y\left(\overline{v_{3}}\right)-y\left(w_{1}^{\prime}\right)+1}{x\left(\overline{v_{3}}\right)-x\left(w_{1}^{\prime}\right)-1}-\overline{q_{1}} \\
& =\frac{y\left(\overline{v_{3}}\right)-y\left(w_{1}^{\prime}\right)+1-\left(x\left(\overline{v_{3}}\right)-x\left(w_{1}^{\prime}\right)\right) \overline{q_{1}}+\overline{q_{1}}}{x\left(\overline{v_{3}}\right)-x\left(w_{1}^{\prime}\right)-1}=\frac{1+\overline{q_{1}}}{x\left(\overline{v_{3}}\right)-x\left(w_{1}^{\prime}\right)-1}=\frac{1+\overline{q_{1}}}{x\left(v_{3}\right)-x\left(v_{1}\right)-1} \\
& \leq \frac{1+\overline{q_{1}}}{x\left(z_{\rho(i)}\right)-x\left(b_{1}\right)-1} \leq \frac{1+\overline{q_{1}}}{\alpha-1}=\frac{1+q_{1}+\varepsilon}{\alpha-1} \leq \frac{1+M+\varepsilon}{\alpha-1} \leq \frac{1+M+(i-1)}{\alpha-1} \\
& \leq \frac{2 n^{2}+n}{\alpha-1}=1 .
\end{aligned}
$$

In the third line we have used the fact that $x\left(v_{3}\right)-x\left(v_{1}\right) \geq x\left(z_{\rho(i)}\right)-x\left(b_{1}\right)$, which we demonstrated above in order to establish $\mathcal{P}(i, 1)$. An analogous calculation shows that $\overline{q_{2}}-q_{2}^{\prime}<1$.

Since $a_{i}^{\prime}=\left(\left\lfloor x\left(\overline{v_{3}}\right)\right\rfloor,\left\lceil y\left(\overline{v_{3}}\right)\right\rceil\right)$, the vertex $a_{i}^{\prime}$ lies above and to the left of $\overline{v_{3}}$. Therefore we clearly have $q_{1}^{\prime}-\overline{q_{1}} \geq 0$ and $\overline{q_{2}}-q_{2}^{\prime} \geq 0$. We may now compute

$$
\left|q_{1}^{\prime}-q_{1}\right| \leq\left|q_{1}^{\prime}-\overline{q_{1}}\right|+\left|\overline{q_{1}}-q_{1}\right|=\left|q_{1}^{\prime}-\overline{q_{1}}\right|+\varepsilon<1+\varepsilon \leq 1+(i-1)=i .
$$

An identical calculation shows that $\left|q_{2}^{\prime}-q_{2}\right| \leq i$. Note that $q_{1}^{\prime}$ is the slope of the boundary edge $w_{1}^{\prime} a_{i}^{\prime}$ of $G_{i}^{\prime}$ and $q_{1}$ is the slope of the edge $b_{1} z_{\rho(i)}=Z\left(w_{1}^{\prime} a_{i}^{\prime}\right)$, and similarly for $q_{2}^{\prime}$ and $q_{2}$. This establishes $\mathcal{P}(i, 2)$.

From the construction of $a_{i}^{\prime}$ it is clear that the line segments $w_{1}^{\prime} a_{i}^{\prime}$ and $a_{i}^{\prime} w_{2}^{\prime}$ intersect $G_{i-1}^{\prime}$ only in the vertices $w_{1}^{\prime}$ and $w_{2}^{\prime}$. Thus $G_{i}^{\prime}$ is a plane triangulation, and $G_{i}^{\prime} \sim G_{i}$. To show that $\mathbf{F}\left(G_{i}^{\prime}\right)$ is projectively convex, we proceed similarly to the $d_{i}\left(a_{i}\right)>2$ case.

Let $\hat{q_{1}}$ denote the slope of the boundary edge of $G_{i}^{\prime}$ adjacent and to the left of $w_{1}^{\prime}$, if such an edge exists, and let $\hat{q_{2}}$ denote the slope of the boundary edge of $G_{i}^{\prime}$ adjacent and to the right of $w_{2}^{\prime}$, if such an edge exists. Let $Z\left(\hat{q_{1}}\right)$ and $Z\left(\hat{q_{2}}\right)$ denote the boundary slopes of $Z_{\rho(i-1)}$ corresponding to $\hat{q_{1}}$ and $\hat{q_{2}}$, respectively. By $\mathcal{P}(i-1,2)$, we have $\overline{q_{1}}-q_{1}=\epsilon \leq i-1$ and $Z\left(\hat{q_{1}}\right)-\hat{q_{1}} \leq i-1$. Therefore, by a calculation identical to that in the $d_{i}\left(a_{i}\right)>2$ case (see inequality (3.1)), we conclude that $\hat{q_{1}}-q_{1}^{\prime}>0$ and $q_{2}^{\prime}-\hat{q_{2}}>0$.

Thus $w_{1}^{\prime}$ and $w_{2}^{\prime}$ are convex vertices of $G_{i}^{\prime}$. Because the region $\mathbf{F}\left(G_{i-1}^{\prime}\right)$ is projectively convex by $\mathcal{P}(i-1,3)$, we conclude that $\mathbf{F}\left(G_{i}^{\prime}\right)$ is projectively convex. By $\mathcal{P}(i-1,3)$, the sequence $\left(a_{1}^{\prime}, \ldots, a_{i-1}^{\prime}\right)$ is a convex shedding sequence for $G_{i-1}^{\prime}$, hence $\left(a_{1}^{\prime}, \ldots, a_{i}^{\prime}\right)$ is a convex 
shedding sequence for $G_{i}^{\prime}$. Thus $\mathcal{P}(i, 3)$ holds. We have now established $\mathcal{P}(i)$ in the case that $d_{i}\left(a_{i}\right)=2$.

This completes the induction, and we conclude that $\mathcal{P}(i)$ holds for all $3 \leq i \leq n$. Thus the triangulation $G^{\prime}=G_{n}^{\prime}$ is a sequentially convex embedding of $G$, with convex shedding sequence $\mathbf{a}^{\prime}=\left(a_{1}^{\prime}, \ldots, a_{n}^{\prime}\right)$.

We have immediately that the $x$ dimension of $G^{\prime}$ is at most

$$
\alpha 2(n-2)=\left(2 n^{2}+n+1\right)(2 n-4)=4 n^{3}-6 n^{2}-2 n-4 \leq 4 n^{3} .
$$

Since $\mathcal{P}(n, 2)$ holds, we conclude that the largest absolute value of a boundary slope of $G^{\prime}$ is at most $M+n \leq 2 n^{2}+n$. Thus the $y$ dimension of $G^{\prime}$ is at most

$\alpha 2(n-2)\left(2 n^{2}+n\right)=\left(4 n^{3}-6 n^{2}-2 n-4\right)\left(2 n^{2}+n\right)=8 n^{5}-8 n^{4}-10 n^{3}-10 n^{2}-4 n \leq 8 n^{5}$.

Therefore $G^{\prime}$ is embedded in a $4 n^{3} \times 8 n^{5}$ integer grid.

\section{The Shedding DiAmeter}

Let $G=(V, E)$ be a plane triangulation and let $\mathcal{A}_{G}$ denote the set of all shedding sequences for $G$. For $\mathbf{a}=\left(a_{1}, \ldots, a_{n}\right) \in \mathcal{A}_{G}$, we write $a_{j} \rightarrow_{\mathbf{a}} a_{i}$ if $a_{j}$ is adjacent to $a_{i}$ in $G_{i}(\mathbf{a})$. Then we define the height of each vertex $a_{i}$ recursively, by

$$
\tau\left(a_{i}, \mathbf{a}\right)= \begin{cases}i & i \leq 3 \\ 1+\max \left\{\tau\left(a_{j}, \mathbf{a}\right) \mid a_{j} \rightarrow_{\mathbf{a}} a_{i}\right\} & i>3\end{cases}
$$

We define the height of the shedding sequence $\mathbf{a} \in \mathcal{A}_{G}$ by

$$
\tau(\mathbf{a})=\max _{i} \tau\left(a_{i}, \mathbf{a}\right)
$$

and the shedding diameter of $G$ by

$$
\tau(G)=\min _{\mathbf{a} \in \mathcal{A}_{G}} \tau(\mathbf{a}) .
$$

Taking the transitive closure of the relation $\rightarrow_{\mathbf{a}}$, we obtain a partial order $\preceq \mathbf{a}$ on the vertices of $G$. The height $\tau(\mathbf{a})$ of the sequence $\mathbf{a}$ is then precisely the height of $\preceq_{\mathbf{a}}$. That is, $\tau(\mathbf{a})$ is the maximal length of a chain in $\preceq \mathbf{a}$. One way to visualize this is to direct the edges of $G$ from vertices of lower index in a to vertices of higher index. Then $\tau(\mathbf{a})$ is the maximal length of a directed path.

The next lemma involves the following intuitive notion. Let $\pi: \mathbb{R}^{3} \rightarrow \mathbb{R}^{2}$ denote the coordinate projection $\pi(x, y, z)=(x, y)$. We say that a convex polyhedron $P \subset \mathbb{R}^{3}$ (possibly unbounded) projects vertically onto a geometric plane graph $G$, if $\pi(P)=\mathbf{F}(G)$, and $\pi$ induces an isomorphism on the face structures of $P$ and $G$. This last condition means that $w_{1}, \ldots, w_{k}$ are the vertices of a facet (2-face) of $P$ if and only if $\pi\left(w_{1}\right), \ldots, \pi\left(w_{k}\right)$ are the vertices of a face of $G$.

Lemma 4.1 Let $G$ be a plane triangulation with $n$ vertices and shedding sequence $\boldsymbol{a} \in \mathcal{A}_{G}$, embedded as in Theorem 3.3, so that $\boldsymbol{a}$ is a convex shedding sequence for $G$. Then there is a convex polyhedron $P_{i}$ that projects vertically onto $G_{i}$, for each $i=3, \ldots, n$. Furthermore, if $h\left(a_{i}\right)$ denotes the height of the vertex of $P_{i}$ projecting to $a_{i}$, then we may choose $h\left(a_{i}\right)$ to be an integer such that $h\left(a_{i}\right) \leq 499 n^{8} m_{i}+1$, where

$$
m_{i}=\max \left\{h\left(a_{j}\right) \mid a_{j} \rightarrow_{a} a_{i}\right\} .
$$


Proof. We proceed by induction on $i$. Let $h(v)$ denote the height assigned to the vertex $v \in V(G)$, and let $\varphi(v)=(x(v), y(v), h(v)) \in \mathbb{R}^{3}$ denote the point of $\mathbb{R}^{3}$ projecting vertically to $v$. We define $h\left(a_{1}\right)=h\left(a_{2}\right)=h\left(a_{3}\right)=0$, and let

$$
P_{3}=\left\{(x, y, z) \in \mathbb{R}^{3} \mid(x, y) \in \operatorname{conv}\left(a_{1}, a_{2}, a_{3}\right), z \geq 0\right\} .
$$

That is, $P_{3}$ is the unbounded prism with triangular face $a_{1} a_{2} a_{3}$ and lateral edges extending in the positive vertical direction, parallel to the $z$-axis.

If $i>3$, then by the induction hypothesis, there is a convex polyhedron $P_{i-1}$ that projects vertically onto $G_{i-1}$. So in particular, the vertices of $P_{i-1}$ are $\varphi\left(a_{1}\right), \varphi\left(a_{2}\right), \ldots, \varphi\left(a_{i-1}\right)$. To obtain a lifting of $G_{i}$, we must choose $h\left(a_{i}\right)$ properly. Namely, we must choose $h\left(a_{i}\right)$ large enough to ensure that $\varphi\left(a_{i}\right)$ is in convex position with respect to $\varphi\left(a_{1}\right), \varphi\left(a_{2}\right), \ldots, \varphi\left(a_{i-1}\right)$.

Let $\mathcal{S}_{i}$ denote the set of faces of $G_{i-1}$ having a vertex $v$ such that $v \rightarrow_{\mathbf{a}} a_{i}$, and let $\varphi\left(\mathcal{S}_{i}\right)$ denote the facets of $P_{i-1}$ that project vertically to the faces of $\mathcal{S}_{i}$. We choose the height $h\left(a_{i}\right)$ large enough so that for every facet $F \in \varphi\left(\mathcal{S}_{i}\right)$, the point $\varphi\left(a_{i}\right)$ lies above the hyperplane spanned by $F$. That is, we require that $\varphi\left(a_{i}\right)-(0,0, k)$ is coplanar with $F$ for some $k>0$.

Let $\ell_{i}$ denote the ray with vertex $\varphi\left(a_{i}\right)$ and extending in the positive vertical direction, parallel to the $z$-axis. Then we define a convex polyhedron $P_{i}=\operatorname{conv}\left(P_{i-1} \cup \ell_{i}\right)$. By the choice of $h\left(a_{i}\right)$, the point $\varphi\left(a_{i}\right)$ lies above all facet hyperplanes of $\varphi\left(\mathcal{S}_{i}\right)$, hence above all facet hyperplanes of $P_{i-1}$. Thus the vertices of $P_{i}$ are $\varphi\left(a_{1}\right), \varphi\left(a_{2}\right), \ldots \varphi\left(a_{i}\right)$, and $\varphi\left(a_{i}\right)$ is not a vertex of any facet of $P_{i-1}$. This last fact implies, because $G_{i}$ is a triangulation, that all faces in $\mathcal{F}\left(G_{i}\right) \backslash \mathcal{F}\left(G_{i-1}\right)$ are obtained from the projection of the new facets of $P_{i}$. On the other hand, because $\mathbf{F}\left(G_{i}\right)$ is convex, all new facets of $P_{i}$ project vertically to faces in $\mathcal{F}\left(G_{i}\right) \backslash \mathcal{F}\left(G_{i-1}\right)$. Since $P_{i-1}$ projects vertically onto $G_{i-1}$, these last two statements imply that $P_{i}$ projects vertically onto $G_{i}$.

Now we determine an upper bound on the height $h\left(a_{i}\right)$ necessary for the above construction. To do this, we determine an upper bound on the coordinate $z$ for which $\left(x\left(a_{i}\right), y\left(a_{i}\right), z\right)$ is coplanar with some facet in $\varphi\left(\mathcal{S}_{i}\right)$. If we take $h\left(a_{i}\right)$ to be any integer greater than this upper bound, then $\varphi\left(a_{i}\right)$ lies above the hyperplane of every facet in $\varphi\left(\mathcal{S}_{i}\right)$.

We write $x_{0}=x\left(a_{i}\right), y_{0}=y\left(a_{i}\right)$, and let $z_{0}>0$. Fix $F \in \mathcal{S}_{i}$ and let $v_{1}, v_{2}, v_{3}$ denote the vertices of $F$. Let $\left(x_{j}, y_{j}, z_{j}\right) \in \mathbb{R}^{3}$ denote the coordinates of $\varphi\left(v_{j}\right)$. So in particular $\left(x_{0}, y_{0}\right)=a_{i}$, and $\left(x_{j}, y_{j}\right)=v_{j}$ for $j=1,2,3$.

Suppose that $\varphi\left(a_{i}\right)=\left(x_{0}, y_{0}, z_{0}\right)$ is coplanar with $\varphi\left(v_{1}\right), \varphi\left(v_{2}\right), \varphi\left(v_{3}\right)$. Then $\varphi\left(a_{i}\right)$ is an affine combination of $\varphi\left(v_{1}\right), \varphi\left(v_{2}\right), \varphi\left(v_{3}\right)$. That is, there are scalars $c_{1}, c_{2}, c_{3}$ such that

$$
\left(\begin{array}{ccc}
x_{1} & x_{2} & x_{3} \\
y_{1} & y_{2} & y_{3} \\
1 & 1 & 1
\end{array}\right)\left(\begin{array}{l}
c_{1} \\
c_{2} \\
c_{3}
\end{array}\right)=\left(\begin{array}{c}
x_{0} \\
y_{0} \\
1
\end{array}\right)
$$

and

$$
z_{0}=c_{1} z_{1}+c_{2} z_{2}+c_{3} z_{3}
$$

Let $A$ denote the matrix on the left side of (4.1). By Cramer's rule, $c_{i}=\frac{\operatorname{det}\left(A_{i}\right)}{\operatorname{det}(A)}$, where $A_{i}$ is obtained by replacing the $i^{\text {th }}$ column of $A$ with $\left(x_{0}, y_{0}, 1\right)^{T}$.

Since $G$ is embedded as in Theorem 3.3, the vertices of $G$ lie in a $4 n^{3} \times 8 n^{5}$ integer grid. Furthermore, from the construction of Theorem 3.3, the point $(0,0)$ is contained in the edge $a_{1} a_{2}$ of $G$. This implies that $\left|x_{i}\right| \leq 4 n^{3}$ and $\left|y_{i}\right| \leq 8 n^{5}$ for $i=0,1,2,3$. Therefore, using 
Hadamard's inequality, we obtain

$$
\left|\operatorname{det}\left(A_{i}\right)\right| \leq\left(\sqrt{3} \max _{0 \leq i \leq 3}\left|x_{i}\right|\right)\left(\sqrt{3} \max _{0 \leq i \leq 3}\left|y_{i}\right|\right)\|(1,1,1)\| \leq\left(4 n^{3} \sqrt{3}\right)\left(8 n^{5} \sqrt{3}\right)(\sqrt{3})=96 \sqrt{3} n^{8}
$$

for $i=1,2,3$.

Since $A$ is an invertible integer matrix, we have $|\operatorname{det}(A)| \geq 1$. Thus

$$
\left|c_{i}\right|=\frac{\left|\operatorname{det}\left(A_{i}\right)\right|}{|\operatorname{det}(A)|} \leq\left|\operatorname{det}\left(A_{i}\right)\right| \leq 96 \sqrt{3} n^{8} .
$$

for $i=1,2,3$. Then from (4.2), we have

$$
z_{0} \leq 3\left(\max _{1 \leq i \leq 3}\left|c_{i}\right|\right)\left(\max _{1 \leq i \leq 3}\left|z_{i}\right|\right) \leq 3\left(96 \sqrt{3} n^{8}\right) m_{i} .
$$

So letting $z_{0}$ be the smallest integer greater than $499 n^{8} m_{i}$ will ensure that $\left(x_{0}, y_{0}, z_{0}\right)$ lies above the hyperplane containing $\varphi\left(v_{1}\right), \varphi\left(v_{2}\right), \varphi\left(v_{3}\right)$. Thus we may take $h\left(a_{i}\right) \leq 499 n^{8} m_{i}+1$, as desired.

Theorem 4.2 Let $G$ be a plane triangulation with $n$ vertices. Then $G$ is the vertical projection of a convex 3 -polyhedron with vertices lying in a $4 n^{3} \times 8 n^{5} \times\left(500 n^{8}\right)^{\tau(G)}$ integer grid.

Proof. Choose a shedding sequence $\mathbf{a} \in \mathcal{A}_{G}$ such that $\tau(G)=\tau(\mathbf{a})$. By Theorem 3.3, we may embed $G$ in a $4 n^{3} \times 8 n^{5}$ integer grid such that $\mathbf{a}=\left(a_{1}, \ldots, a_{n}\right)$ is a convex shedding sequence for $G$. For each vertex $a_{i}$ we may assign a height $h\left(a_{i}\right)$ as follows. For $i=1,2,3$ we may set $z_{i}=0$. For $i>3$, by Lemma 4.1 we may choose $h\left(a_{i}\right)$ such that $G_{i}$ is the projection of a polyhedral surface, and

$$
h\left(a_{i}\right) \leq\left(499 n^{8}+1\right)^{\tau\left(a_{i}, \mathbf{a}\right)} \leq\left(500 n^{8}\right)^{\tau\left(a_{i}, \mathbf{a}\right)} \leq\left(500 n^{8}\right)^{\tau(\mathbf{a})}=\left(500 n^{8}\right)^{\tau(G)} .
$$

Note that if the boundary of $G$ is a triangle (that is, $\partial \mathbf{F}(G)$ contains exactly three vertices), then the polyhedron of Theorem 4.2 may be replaced with a (bounded) 3-polytope. Indeed, simply truncate the polyhedron with the hyperplane that is defined by the lifts of the three boundary vertices of $G$. Then the three boundary vertices of $G$ lift to the vertices of a triangular face of the resulting 3-polytope.

\section{Triangulations of a ReCtangular Grid}

For $p, q \in \mathbb{Z}, p, q \geq 2$, let $[p \times q]=\{1, \ldots, p\} \times\{1, \ldots, q\}$. We may think of the integer lattice $[p \times q]$ as the vertices of $(p-1)(q-1)$ unit squares. A geometric plane triangulation $G$ is a triangulation of $[p \times q]$ if the vertices of $G$ are exactly the vertices of $[p \times q]$, and every boundary edge of $[p \times q]$ is an edge of $G$. We call $G$ a grid triangulation. An $\ell \times \ell$ subgrid of $\mathbb{Z}^{2}$ is an integer translation of the lattice $[\ell \times \ell]=\{1, \ldots, \ell\} \times\{1, \ldots, \ell\}$. By an $\ell \times \ell$ subgrid of $[p \times q]$ we mean an $\ell \times \ell$ subgrid of $\mathbb{Z}^{2}$ that is a subset of $[p \times q]$. In this section we state and prove the following result concerning the shedding diameter of grid triangulations.

Theorem 5.1 Let $G$ be a triangulation of $[p \times q]$ such that every edge $e$ of $G$ sits in an $\ell \times \ell$ subgrid. Then $\tau(G) \leq 6 \ell(p+q)$. 
This gives a class of triangulations with sublinear shedding diameter, if $\ell$ is held constant. According to Theorem 4.2, such a triangulation can be drawn in the plane so that it is the vertical projection of a simplicial 3-polyhedron embedded in a subexponential grid. That is, this class of triangulations corresponds to a class of simplicial polyhedra which may be embedded in an integer grid whose size is subexponential in the number of vertices.

Let $\leq_{\mathbb{Z}^{2}}$ denote the linear order on $\mathbb{Z}^{2}$ defined by

$$
\left(x_{1}, y_{1}\right) \leq_{\mathbb{Z}^{2}}\left(x_{2}, y_{2}\right) \text { if and only if } y_{1}<y_{2} \text {, or } y_{1}=y_{2} \text { and } x_{1} \leq x_{2} .
$$

That is, $\leq_{\mathbb{Z}^{2}}$ is a lexicographic order in which $y$-coordinates take precedence in determining the order. We state without proof the following lemma, which summarizes some standard properties of shedding vertices of planar triangulations (see [BP, §3] for a proof and references). Recall from Section 2 that $\mathbf{F}(G) \subset \mathbb{R}^{2}$ is homeomorphic to a ball. Therefore if $e$ is a diagonal of $\mathbf{F}(G)$, then the set $\mathbf{F}(G) \backslash e$ has two connected components.

Lemma $5.2([\mathrm{BP}])$ Let $G$ be a plane triangulation, and let $v$ be a boundary vertex of $G$. Then either $v$ is a shedding vertex of $G$, or it is the endpoint of a diagonal e of $G$. In the latter case, each of the two connected components of $\mathbf{F}(G) \backslash e$ contains a shedding vertex of $G$.

The rough idea of the proof of Theorem 5.1 is as follows (we provide the details below). We begin by constructing a particular shedding sequence a for $G$. To do this, we first subdivide $[p \times q]$ into a grid of $\left\lceil\frac{p q}{\ell^{2}}\right\rceil$ subgrids, (most of) which are squares of size $\ell \times \ell$. These squares form $\left\lceil\frac{p}{\ell}\right\rceil$ columns and $\left\lceil\frac{q}{\ell}\right\rceil$ rows.

We shed $G$ in three stages. In Stage 1, we take every fourth column $U(1), U(5), U(9), \ldots$ and shed the vertices of each of these columns from top to bottom. When shedding $U(i)$, we may need to shed vertices in the column $U(i-1)$ or $U(i+1)$, for a total of at most $3 q \ell$ vertices shed in the process of shedding the column $U(i)$. Because of their spacing, the shedding vertices in each column do not interact. Specifically, at each step we have a collection of shedding vertices, one from each column, which we may think of as shedding "all at once". This collection of vertices is then an antichain with respect to $\preceq_{\mathbf{a}}$. When shedding the vertices of each such column, to maintain connectivity we do not shed the vertices $(x, y)$ with $y \leq \ell$. See Figure 6 .

After Stage 1 is complete, what remains are a set of "jagged tricolumns", each of which consists of the remaining vertices of three adjacent columns. Hence each jagged tricolumn contains at most $3 q \ell$ vertices. In Stage 2 , we shed these columns, but to maintain connectivity, we do not shed vertices $(x, y)$ with $y \leq 2 \ell$. As before, these jagged tricolumns do not interact, and at each step we have a set of shedding vertices, each of which belongs to a different jagged tricolumn. Hence this set forms an antichain. Finally, in Stage 3 we shed the remaining vertices, which are contained in the bottom two rows of $G$. There are at most $2 p \ell$ such vertices, and we simply define a singleton antichain for each of them. Therefore we see that $G$ may be partitioned into at most $2 p \ell+3 q \ell+3 q \ell=\ell(2 p+6 q) \leq 6 \ell(p+q)$ antichains of $\preceq \mathbf{a}$. This implies that $\tau(\mathbf{a}) \leq 6 \ell(p+q)$, since $\tau(\mathbf{a})$ is the length of some chain in $\preceq \mathbf{a}$. The detailed proof follows.

Proof of Theorem 5.1. Let $G$ be such a triangulation of $[p \times q]$. For $i \in \mathbb{Z}$, let

$$
\begin{aligned}
U(i) & =\{(x, y) \in[p \times q] \mid \ell(i-1)+1 \leq x \leq \ell i\}, \quad \text { and } \\
R & =\{(x, y) \in[p \times q] \mid 1 \leq y \leq \ell\} .
\end{aligned}
$$




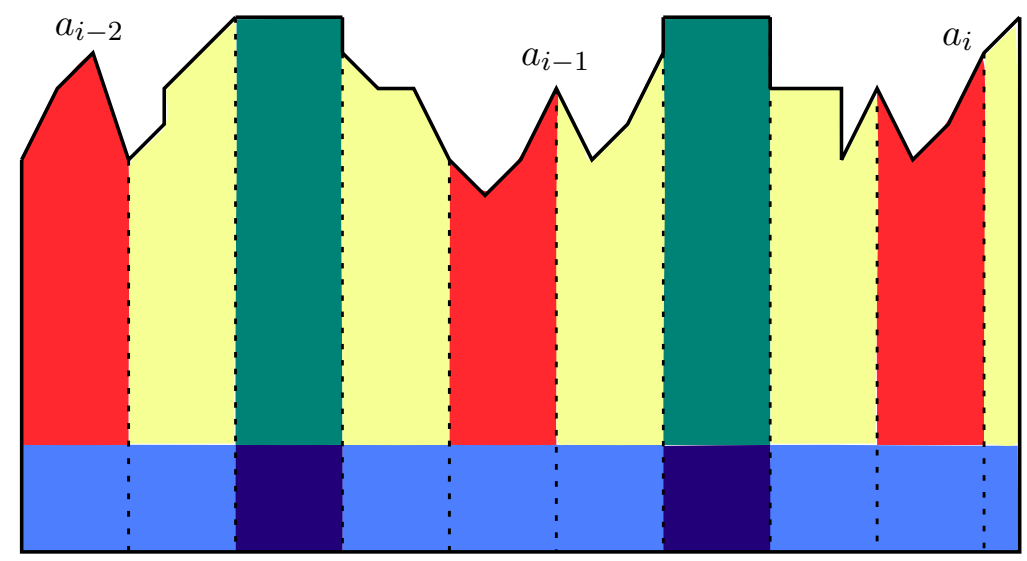

Figure 6. A graph $G_{i}$ produced during Stage 1 of the construction of a in the proof of Theorem 5.1. Distinct columns $U(j)$ are separated by dashed lines. The columns of the form $U(1+4 j)$ are shown in red, while the columns $U(3+4 j)$ are shown in green. The bottom row $R$ is shown in blue.

Many of these sets are empty (for example when $i \leq 0$ ). We think of the sets $U(i)$ as columns of width $\ell$ and $R$ as the bottom row of height $\ell$. For each $i \in \mathbb{Z}$, we also define

$$
T(i)=U(i-1) \cup U(i) \cup U(i+1),
$$

which may be empty. We call $T(i)$ a tricolumn of $[p \times q]$.

We construct the shedding sequence a recursively. Suppose that we have a sequence of shedding vertices $a_{i+1}, a_{i+2}, \ldots, a_{n}$ (for the initial step of the recursion, $i=n$ and this sequence is empty), and therefore we also have plane triangulations $G_{i}, G_{i+1}, \ldots, G_{n}=G$, where as usual $G_{j-1}=G_{j}-\left\{a_{j}\right\}$ for all $j=i+1, \ldots, n$. For each $i=1, \ldots, n$, let $R_{i}$ denote the subgraph of $G_{i}$ induced by the vertices in $R$. Similarly, for each $i=1, \ldots, n$ and $j \in \mathbb{Z}$, let $U_{i}(j)$ denote the subgraph of $G_{i}$ induced by the vertices in $U(j)$. We let $\mathcal{P}(i, 1), \mathcal{P}(i, 2)$, and $\mathcal{P}(i, 3)$ denote the following statements:

$$
\begin{array}{ll}
\mathcal{P}(i, 1) . & U_{i}(3+4 j)=U_{n}(3+4 j) \\
\mathcal{P}(i, 2) . & R_{i} \text { is connected. }
\end{array}
$$

Note that $\mathcal{P}(n, 1)$ holds trivially. Furthermore, we have $G_{n}=G$, so the vertices of $U_{n}(1+3 j)$ are exactly those of $U(1+3 j)$, and similarly for $R_{n}$ and $R$. Since $G$ is a grid triangulation, it follows that $U_{n}(1+3 j)$ and $R_{n}$ are connected. In particular, $\mathcal{P}(n, 2)$ holds.

To construct the vertex $a_{i}$ of the shedding sequence, we break the construction into three stages, described below. As can readily be seen, each stage occurs for a consecutive sequence of indices. That is, there are integers $i_{2}<i_{1}$ such that Stage 1 occurs for $i=i_{1}, i_{1}+1, \ldots, n$, Stage 2 occurs for $i=i_{2}, i_{2}+1, \ldots, i_{1}-1$, and Stage 3 occurs for $i=1,2, \ldots, i_{2}-1$. We will also show, as we describe these stages, that $\mathcal{P}(i, 1)$ and holds for $i=i_{1}, i_{1}+1, \ldots, n$, and $\mathcal{P}(i, 2)$ holds for $i=i_{2}, i_{2}+1, \ldots, i_{1}-1$. That is, $\mathcal{P}(i, 1)$ holds through all of Stage 1 , and $\mathcal{P}(i, 2)$ holds through all of Stage 2. Furthermore, during Stages 1 and 2, we will never remove vertices $v$ with $y(v)=1$. We will use this fact when showing that $\mathcal{P}(i, 1)$ holds.

At the beginning of the section for each stage, we state the condition that uniquely determines the stage. The stage ends when its condition is no longer satisfied.

Stage 1. Some column of the form $U(1+4 j)$ contains a vertex $(x, y)$ of $G_{i}$ with $y>\ell$. 
Let $U\left(1+4 j_{1}\right), \ldots, U\left(1+4 j_{r}\right)$ denote all such columns, where $j_{1}<\cdots<j_{r}$. See Figure 6 . Assume that $\mathcal{P}(i, 1)$ and $\mathcal{P}(i, 2)$ hold.

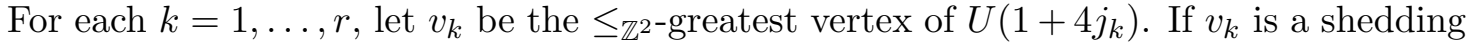
vertex of $G_{i}$, define $w_{k}=v_{k}$.

Otherwise, by Lemma 5.2, the vertex $v_{k}$ is the endpoint of a diagonal of $G_{i}$. Let $u_{k}$

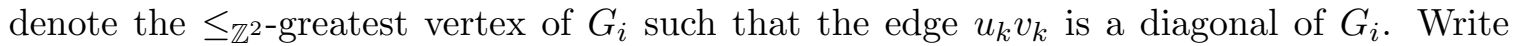
$e_{k}=u_{k} v_{k}$. Then $\mathbf{F}\left(G_{i}\right) \backslash e_{k}$ has two connected components, call them $A_{k}$ and $A_{k}^{\prime}$. Since the vertices $u_{k}$ and $v_{k}$ are adjacent, by assumption they are contained in an $\ell \times \ell$ subgrid of $[p \times q]$. It follows that $u_{k} \in T\left(1+4 j_{k}\right)$. Thus $u_{k}, v_{k} \notin U(3+4 j)$ for all $j$.

As mentioned above, the column $U_{n}(3+4 j)$ is connected for all all $j$. So by $\mathcal{P}(i, 1)$, $U_{i}\left(3+4 j_{k}\right)$ is connected. We also have $y\left(v_{k}\right)>\ell$, and thus $y\left(u_{k}\right)>1$, which implies that $A_{k}$ does not intersect the line segment $[1, p] \times\{1\}$ (which consists of the bottom vertices and edges of $R$ ). But each column $U_{i}(3+4 j)$ clearly intersects this line segment. Therefore one of the components of $\mathbf{F}\left(G_{i}\right) \backslash e_{k}$, say $A_{k}$, does not intersect $U_{i}(3+4 j)$ for all $j$.

It follows that all vertices in $A_{k}$ are contained in $T\left(1+4 j_{k}\right)$. By Lemma 5.2, the region

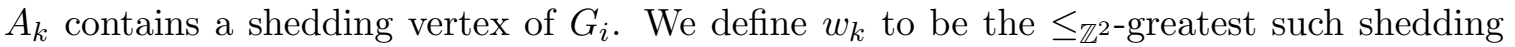
vertex.

We now have a collection of shedding vertices $w_{1}, \ldots, w_{r}$ of $G_{i}$. Clearly the neighbors of each vertex $w_{k}$ lie in the tricolumn $T\left(1+4 j_{k}\right)$, so no two of the vertices $w_{1}, \ldots w_{r}$ are adjacent to a common vertex. Thus the vertex $w_{r-1}$ is a shedding vertex of $G_{i}-\left\{w_{r}\right\}$, the vertex $w_{r-2}$ is a shedding vertex of $G_{i}-\left\{w_{r}, w_{r-1}\right\}$, etc. That is, these vertices remain shedding vertices after deleting any finite subset of them from $G_{i}$. So for each $k=1, \ldots, r$, we may define $a_{i-r+1}, \ldots, a_{i}$ by $a_{i-r+k}=w_{k}$. Since no two of the vertices $a_{i-r+1}, \ldots a_{i}$, are adjacent, the set $\left\{a_{i-r+1}, \ldots, a_{i}\right\}$ is an antichain of $\preceq_{\mathbf{a}}$.

Finally, we must show inductively that the property $\mathcal{P}(i-k, 1)$ holds for all $k=1, \ldots, r$, but this is clear because $w_{k} \notin U(3+4 j)$ for all $j$. This completes Stage 1 .

Before we begin Stage 2, we must show that $\mathcal{P}(i, 2)$ will hold when we begin. Letting $i_{1}$ denote the last step of Stage 1 as in the notation above, this is the claim that $\mathcal{P}\left(i_{1}-1,2\right)$ holds. This will follow directly from the fact that each region $A_{k}$ arising in Stage 1 is shed entirely.

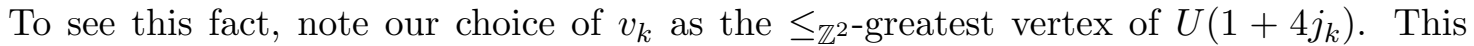
means that, so long as $v_{k}$ is contained in a diagonal of $G_{i}$, we will continue to pick the same vertex $v_{k}$ at each step, finding a new shedding vertex in the same original set $A_{k}$. Once $A_{k}$ is empty, $v_{k}$ will no longer be a diagonal of $G_{i}$, and then we will finally take $w_{k}=v_{k}$ as the shedding vertex of $G_{i}$.

As mentioned above, $R_{n}=R$ is connected. Now suppose inductively that $R_{i}$ is connected, and consider a path $\gamma$ in $R_{i}$ containing $w_{k}$ but with endpoints not in $A_{k}$. Since $y\left(v_{k}\right)>\ell$, this path must enter and exit $A_{k}$ through $u_{k}$. But then $\gamma$ may be replaced with a path $\gamma^{\prime} \subset R_{i} \backslash A_{k}$, having the same endpoints. It follows that $R_{i} \backslash A_{k}$ is connected. Using the above fact that all vertices of $A_{k}$ are shed during Stage 1, we conclude that at the beginning of Stage $2, R_{i}$ is connected. That is, $\mathcal{P}\left(i_{1}-1,2\right)$ holds.

Stage 2. No column of the form $U(1+4 j)$ contains vertices $(x, y)$ of $G_{i}$ with $y>\ell$, but $G_{i}$ contains at least one vertex $(x, y)$ with $y>2 \ell$.

From the criteria for this stage, the vertices $(x, y)$ with $y>2 \ell$ must be contained in a tricolumn of the form $T(3+4 j)$. Let $T\left(3+4 j_{1}\right), \ldots, T\left(3+4 j_{r}\right)$ denote all such tricolumns, where $j_{1}<\cdots<j_{r}$. Assume that $\mathcal{P}(i, 2)$ holds. For each $k=1, \ldots, r$, let $v_{k}$ be the $\leq_{\mathbb{Z}^{2-}}$ greatest vertex of $T\left(3+4 j_{k}\right)$. If $v_{k}$ is a shedding vertex of $G_{i}$, define $w_{k}=v_{k}$. Otherwise, by 

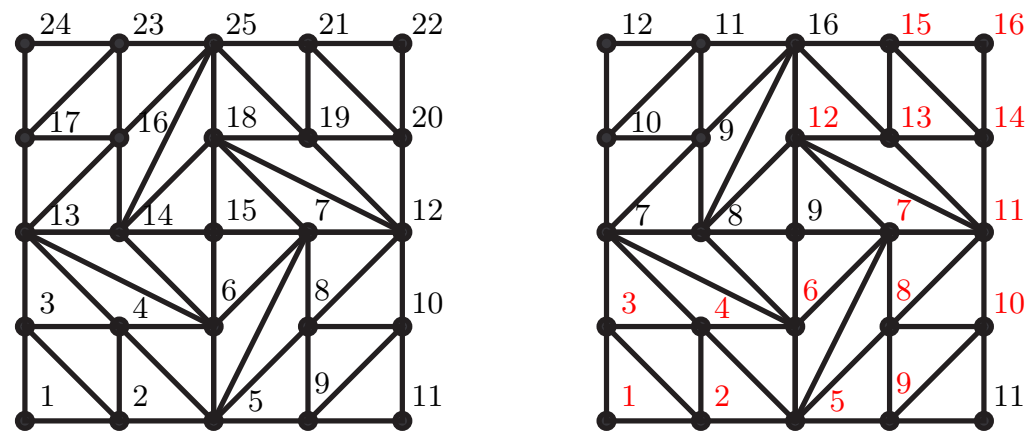

Figure 7 . The grid triangulation of Figure 1 . Here $p=q=5$ and $\ell=3$. Each vertex is labeled with its index $i$ in a shedding sequence a (left) and the corresponding value of $\tau\left(a_{i}\right)$ (right). A chain of maximal length $\tau(\mathbf{a})=16$ is shown in red.

Lemma 5.2, the vertex $v_{k}$ is the endpoint of a diagonal of $G_{i}$. Let $u_{k}$ denote the $\leq_{\mathbb{Z}^{2} \text {-greatest }}$ vertex of $G_{i}$ such that the edge $u_{k} v_{k}$ is a diagonal of $G_{i}$. Write $e_{k}=u_{k} v_{k}$.

Then $\mathbf{F}\left(G_{i}\right) \backslash e_{k}$ has two connected components, call them $A_{k}$ and $A_{k}^{\prime}$. Since the vertices $u_{k}$ and $v_{k}$ are adjacent, by assumption they are contained in an $\ell \times \ell$ subgrid of $[p \times q]$. Since $y\left(v_{k}\right)>2 \ell$, it follows that $y\left(u_{k}\right)>\ell$, and thus $u_{k}, v_{k} \notin R$. Then by $\mathcal{P}(i, 2)$, one of the components of $\mathbf{F}\left(G_{i}\right) \backslash e_{k}$, say $A_{k}$, does not intersect $R_{i}$. By definition of Stage 2, we have

$$
V\left(U_{i}(1+4 j)\right) \subseteq R, \quad j \in \mathbb{Z},
$$

so we also conclude that $A_{k}$ does not intersect any column of the form $U(1+4 j)$. By Lemma 5.2, the region $A_{k}$ contains a shedding vertex of $G_{i}$. We define $w_{k}$ to be the

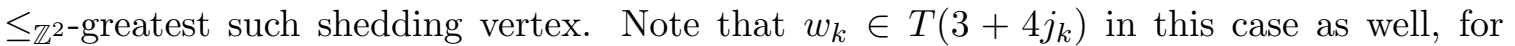
otherwise, either $A_{k}$ contains a vertex in $U\left(1+4 j_{k}\right)$ or $U\left(5+4 j_{k}\right)$, or $G_{i}$ has an edge $u v$ with $|x(u)-x(v)|>\ell$.

We now have a collection of shedding vertices $w_{1}, \ldots, w_{r}$ of $G_{i}$. Every vertex $w_{k}$ lies in the tricolumn $T\left(3+4 j_{k}\right)$, and none of the neighbors of $w_{k}$ are contained in $R$. This implies, by (5.1), that no two of the vertices $w_{1}, \ldots, w_{r}$ are adjacent to a common vertex. Thus these vertices remain shedding vertices after deleting any finite subset of them from $G_{i}$. So for each $k=1, \ldots, r$, we may define $a_{i-r+1}, \ldots, a_{i}$ by $a_{i-r+k}=w_{k}$. Since no two of the vertices $a_{i-r+1}, \ldots a_{i}$, are adjacent, the set $\left\{a_{i-r+1}, \ldots, a_{i}\right\}$ is an antichain of $\preceq \mathbf{a}$.

Finally, note that by construction we have $w_{k} \notin R$ for all $k=1, \ldots, r$. That is, none of the vertices of the row $R$ are deleted in Stage 2. Thus $R_{i-k}=R_{i}$ for all $k=1, \ldots, r$, so from $\mathcal{P}(i, 2)$ we conclude that $\mathcal{P}(i-k, 2)$ holds for all $k=1, \ldots, r$.

Stage 3. All vertices $(x, y)$ of $G_{i}$ have $y \leq 2 \ell$. If $i>3$ we define $a_{i}$ to be the $\leq_{\mathbb{Z}^{2-}}$ greatest shedding vertex of $G_{i}$, which exists by Lemma 2.1. If $i \leq 3$ we define $a_{i}$ to be the

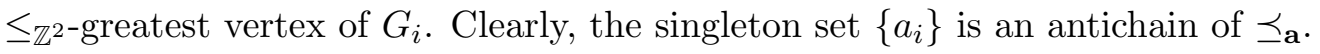

This completes the construction of the shedding sequence $\mathbf{a}=\left(a_{1}, \ldots, a_{n}\right)$ (See Figure 7). It is straightforward to count the number of antichains of $\preceq_{\mathbf{a}}$ obtained from this construction. Stage 1 requires as many steps as it takes for the last column of the form $U(1+4 j)$ to run out of vertices $(x, y)$ with $y>\ell$. Since each vertex $a_{i}$ of Stage 1 is contained in some tricolumn of the form $T(1+4 j)$, this requires at most $|T(1+4 j)|=3 q \ell$ steps, each of which produces an antichain. Similarly, Stage 2 requires as many steps as it takes for the last 
tricolumn of the form $T(3+4 j)$ to run out of vertices $(x, y)$ with $y>2 \ell$. This requires at most $|T(3+4 j)|=3 q \ell$ steps, each of which produces an antichain. Finally, each set $\left\{a_{i}\right\}$ is trivially an antichain, so taking the singleton of each vertex $a_{i}$ defined in Stage 3 yields at most $2 p \ell$ antichains.

The set of antichains of $\preceq \mathbf{a}$ produced by these three cases clearly forms a partition of $V(G)=[p \times q]$. There are at most $2 p \ell+3 q \ell+3 q \ell=\ell(2 p+6 q)$ antichains in this partition. Thus, since $\tau(\mathbf{a})$ is the length of some chain in $\preceq \mathbf{a}$, we have

$$
\tau(G) \leq \tau(\mathbf{a}) \leq \ell(2 p+6 q) \leq 6 \ell(p+q) .
$$

Theorems 4.2 and 5.1 now immediately imply the following general result.

Theorem 5.3 Let $G$ be a grid triangulation of $[p \times q]$ such that every triangle fits in an $\ell \times \ell$ subgrid. Then $G$ can be realized as the graph of a convex polyhedron embedded in an integer grid of size $4(p q)^{3} \times 8(p q)^{5} \times\left(500(p q)^{8}\right)^{6 \ell(p+q)}$.

Corollary 1.2 now follows by setting $p=q=k$.

\section{Final REMARKS AND OPEN PROBlems}

6.1. The study of the Quantitative Steinitz Problem was initiated by Onn and Sturmfels in [OS], who gave the first nontrivial upper bound on the grid size. For plane triangulations, a different approach was given in [DG]. Since then, there have been a series of improvements (see [BS, R, Ro]), leading to the currently best $\exp O(n)$ bound in [RRS]. The only other class of graphs for which there is a known subexponential bound, is the class of triangulations corresponding to stacked polytopes [DS], which can be embedded into a polynomial size grid.

In the opposite direction, there are no non-trivial lower bounds on the size of the grid. If anything, all the evidence suggests that the answer may be either polynomial or nearpolynomial. Note, for example, that while the number of isomorphism classes of simplicial polytopes (which is equal to the number of plane triangulations) on $n$ vertices is $\exp O(n)$ (see e.g. [DRS]), the number of grid polytopes with $n$ vertices in a polynomial size cube $O\left(n^{k}\right) \times O\left(n^{k}\right) \times O\left(n^{k}\right)$ is superexponential, see [BV]. Of course, many of these have isomorphic graphs. In any event, we conjecture that for triangulations a polynomial size grid is sufficient indeed.

6.2. Our Theorem 3.3 is a variation on results in $[\mathrm{BR}, \mathrm{FPP}]$ and can be viewed as a stand alone result in Graph Drawing. It is likely that the polynomial bounds in the theorem can be substantially improved. We refer to [TDET] for general background in the field.

6.3. Let us mention that not every grid triangulation is regular (see [DRS] for definitions and further references). An example found by Santos (quoted in [KZ]), is shown in Figure 1 in the introduction. This means that one cannot lift this triangulation directly to a convex polyhedron; another plane embedding of the triangulation is necessary for that.

6.4. The shedding diameter of a plane triangulation $G$ is closely related and bounded from above (up to an additive constant), by the optimal height of the visibility representation of $G$. This is a parameter of general graphs, defined independently in [RT, TT], and explored extensively in a series of recent papers by He, Zhang and others (see e.g. [HZ, HWZ, ZH1, ZH2]). Motivated by VLSI applications, the results in these papers give linear upper bounds on the optimal height of various classes, which are too weak for the desired subexponential 
upper bounds in the Quantitative Steinitz's Problem. In fact, one can view our Theorem 5.3 as a sublinear bound on the height representation of a class of graphs.

6.5. While the shedding diameter is linear in the worst case, it is sublinear in a number of special cases. For example, for random stacked triangulations the shedding diameter becomes the height of a random ternary tree, or $\Theta(\sqrt{n})$, see e.g. [FS]. For the (nearly-) balanced stacked triangulations $G$ we have $\tau(G)=O(\log n)$, giving a nearly polynomial upper bound in the Quantitative Steinitz Problem. While these cases are covered by a polynomial bound in [DS], notice that our proof is robust enough to generalize to other related iterative families. In fact, we conjecture that $\tau(G)=O(\sqrt{n})$ w.h.p., for random triangulations with $n$ vertices (cf. [CFGN]).

Acknowledgements The authors are grateful to Jesús De Loera, Stefan Felsner, Alexander Gaifullin, János Pach, Rom Pinchasi, Carsten Thomassen, Jed Yang, and Günter Ziegler for helpful comments and interesting conversations. We are especially thankful to Günter Rote for the careful reading of the previous draft of the manuscript, a number of useful remarks and help with the references. A preliminary version of this work has appeared in the second author's Ph.D. thesis [Wi]. The first author was partially supported by the BSF and NSF.

\section{REFERENCES}

[A] E. E. Anclin, An upper bound for the number of planar lattice triangulations, J. Combin. Theory Ser. A 103 (2003), no. 2, 383-386.

[BV] I. Bárány and A. M. Vershik, On the number of convex lattice polytopes, Geom. Funct. Anal. 2 (1992), 381-393.

[BP] L. Billera and J. Provan, Decompositions of simplicial complexes related to diameters of convex polyhedra, Math. Op. Res. 5 (1980), 576-594.

[BR] I. Bárány and G. Rote, Strictly convex drawings of planar graphs, Documenta Math. 11 (2006), 369-391.

[BS] K. Buchin and A. Schulz, On the number of spanning trees a planar graph can have, in Proc. ESA 2010, 576-594.

[CFGN] G. Chapuy, É. Fusy, O. Giménez and M. Noy, On the diameter of random planar graphs, in Proc. 21st AofA, DMTCS, Nancy, 2010, 65-78.

[DG] G. Das and M. T. Goodrich, On the complexity of optimization problems for 3-dimensional convex polyhedra and decision trees, Comput. Geom. Theory Appl. 8 (1997), no. 3, 123-137.

[DRS] J. A. De Loera, J. Rambau and F. Santos, Triangulations: Structures and Algorithms, Springer, 2008.

[DS] E. D. Demaine and A. Schulz, Embedding stacked polytopes on a polynomial-size grid, in Proc. SODA 2011, 1177-1187.

[F] I. Fáry, On straight line representations of planar graphs, Acta Univ. Szeged. Sect. Sci. Math. 11 (1948), 229-233.

[FPP] H. de Fraysseix, J. Pach and R. Pollack, How to draw a planar graph on a grid, Combinatorica 10 (1990), 41-51.

[FS] Ph. Flajolet and R. Sedgewick, Analytic combinatorics, Cambridge University Press, 2009.

[G] B. Grünbaum, Convex polytopes, Springer, Berlin, 1995.

[HWZ] X. He, J.-J. Wang and H. Zhang, Compact visibility representation of 4-connected plane graphs, Theoret. Comput. Sci. 447 (2012), 62-73.

[HZ] X. He and H. Zhang, Nearly optimal visibility representations of plane graphs, SIAM J. Discrete Math. 22 (2008), 1364-1380.

[KZ] V. Kaibel, G. Ziegler, Counting lattice triangulations, in Surveys in combinatorics, 277-307, London Math. Soc. Lecture Note Ser., 307, Cambridge Univ. Press, Cambridge, 2003. 
[L] L. Lovász, Geometric representations of graphs, lecture notes; available electronically at http://www.cs.elte.hu/ lovasz/geomrep.pdf.

[RRS] A. Ribó Mor, G. Rote and A. Schulz, Small grid embeddings of 3-polytopes, Discrete Comput. Geom. 45 (2011), 65-87.

[R] J. Richter-Gebert, Realization spaces of polytopes, Lecture Notes in Math., Springer, 1996.

[RT] P. Rosenstiehl and R. E. Tarjan, Rectilinear planar layouts and bipolar orientations of planar graphs, Discrete Comput. Geom. 1 (1986), 343-353.

[Ro] G. Rote, The number of spanning trees in a planar graph, in Oberwolfach Reports vol. 2, EMS, 2005, 969-973.

[RZ] J. Richter-Gebert and G. M. Ziegler, Realization spaces of 4-polytopes are universal, Bull. Amer. Math. Soc. 32 (1995), 403-412.

[OS] S. Onn and B. Sturmfels, A quantitative Steinitz' theorem, Beiträge zur Algebra und Geometrie 35 (1994), 125-129.

[S] A. Schulz, Drawing 3-polytopes with good vertex resolution, J. Graph Algorithms Appl. 1 (1986), 321-341.

[TT] R. Tamassia and I. G. Tollis, A unified approach to visibility representations of planar graphs, Discrete Comput. Geom. 1 (1986), 321-341.

[TDET] I. G. Tollis, G. Di Battista, P. Eades and R. Tamassia, Graph drawing, Prentice Hall, Upper Saddle River, NJ, 1999.

[T] W. T. Tutte, How to draw a graph, Proc. Lond. Math. Soc. 13 (1963), 743-768.

[We] E. Welzl, The number of triangulations on planar point sets, in Lecture Notes in Comput. Sci. 4372, Springer, Berlin, 2007, 1-4.

[Wi] S. Wilson, Embeddings of Polytopes and Polyhedral Complexes, Ph.D. thesis, UCLA, 2012.

[ZH1] H. Zhang and X. He, Improved visibility representation of plane graphs, Comput. Geom. 30 (2005), 29-39.

[ZH2] H. Zhang and X. He, Optimal st-orientations for plane triangulations, J. Comb. Optim. 17 (2009), 367-377.

[Z1] G. M. Ziegler, Lectures on polytopes, Springer, New York, 1995.

[Z2] G. M. Ziegler, Convex polytopes: extremal constructions and f-vector shapes, in Geometric Combinatorics, AMS, Providence, RI, 2007, 617-691; arXiv: math.MG/0411400. 